UPR-747T, IASSNS-HEP-97/42

hep-th/9704178

April 1997

\title{
U-Duality Symmetries from the Membrane Worldvolume
}

\author{
André Lukas ${ }^{1 *}$ and Burt A. Ovrut ${ }^{1} 2$ \\ ${ }^{1}$ Department of Physics, University of Pennsylvania \\ Philadelphia, PA 19104-6396, USA \\ ${ }^{2}$ School of Natural Sciences, Institute for Advanced Study \\ Olden Lane, Princeton, NJ 08540, USA
}

\begin{abstract}
We study U-duality symmetries of toroidally compactified $M$ theory from the membrane worldvolume point of view. This is done taking the most general set of bosonic background fields into account. Upon restriction to pure moduli backgrounds, we are able to find the correct U-duality groups and moduli coset parameterizations for dimensions $D>6$ as symmetries of the membrane worldvolume theory. In particular, we derive the $D=8 \mathrm{U}$-duality group $S L(2) \times$ $S L(3)$. For general background fields, we concentrate on the case $D=8$. Though the $S L(2)$ part of the symmetry appears to be obstructed by certain terms in the equations of motion, we are able to read off the transformation properties for the background fields. These transformations are verified by comparison with 11-dimensional supergravity dimensionally reduced to $D=8$.
\end{abstract}

*Supported by Deutsche Forschungsgemeinschaft (DFG) and Nato Collaborative Research Grant CRG. 940784. 


\section{Introduction}

Recently, string theory has undergone a dramatic evolution. It is now believed that the perturbative expansions of the five consistent string theories represent patches in the moduli space of a yet larger theory, called M-theory [1]. The low energy limit of this theory is given by 11-dimensional supergravity. Though the correct quantization of $\mathrm{M}$ theory is presently unknown, it is believed to be a theory of fundamental membranes. Correspondingly, the starting point for a quantization of $\mathrm{M}$ theory [2] is the 11-dimensional supermembrane action [3]. Classically, imposing $\kappa$ symmetry on the supermembrane worldvolume restricts the background fields, the metric, a 3-form field and their superpartners, to fulfill the equations of motion of 11-dimensional supergravity [4].

Duality symmetries play an important rôle in this picture in that they relate the various string theories and 11-dimensional supergravity, compactified on different backgrounds, to each other. This provides evidence for the existence of a more general underlying theory. These relations have basically been established by showing that the soliton spectra [5 of certain theories are mapped into each other by duality transformations. Clearly, a major goal is to prove the invariance of the underlying theory, compactified quantum $\mathrm{M}$ theory, under these symmetries. A first step toward such a proof is to analyze to what extent these symmetries are realized in the corresponding classical theory, namely in the 11-dimensional supermembrane. It is this question which we are going to address in the present paper, for the case of $\mathrm{M}$ theory toroidally compactified to $D$ space-time dimensions. The corresponding U-duality symmetries have been known for a long time as classical continuous symmetries of dimensionally reduced 11-dimensional supergravity [6]. Recently, Hull and Townsend [7] found evidence that discrete versions of these continuous U-duality groups are symmetries of the full theory, by showing the invariance of certain BPS soliton spectra. Since these U-duality symmetries include $\mathrm{T}$ as well as S duality, they provide a "unified" picture of dualities. It is the main purpose of this paper to investigate to what extent this unified picture of dualities arises from the worldvolume theory of the 11-dimensional supermembrane. Before explaining this in more detail, however, let us briefly describe the analogous problem in string theory.

$\mathrm{T}$ duality [8] is a well known exact symmetry of perturbative string theory. It can be seen to leave the perturbative spectrum invariant, by exchanging momentum and winding modes, if simultaneously the "radius of compactification" is inverted. Classically, the discrete T-duality symmetry enlarges to a continuous group of $\mathrm{T}$-duality rotations which arise as symmetries of the string $\sigma$ model. For a pure moduli background, this has been shown in ref. [9] by analyzing the symmetries of the combined system of equations of motion and Bianchi identities. This applies a method developed earlier in the context of 4-dimensional gauge theories [10] to the string $\sigma$ model. The generalization to include the full bosonic background field content of the theory was provided in ref. [11]. Duality rotations on the full background field content constitute a generalization of 
the discrete Buscher duality transformation [12]. On the other hand, S duality, as a consequence of string-string duality in six dimensions [13], is a symmetry of string theory in $D=4$ [14, 15]. Since S duality inverts the coupling constant of the theory (the dilaton), it is a nonperturbative symmetry. As such, it does not leave the perturbative spectrum of the theory invariant and its continuous version is, therefore, not a classical symmetry of the string $\sigma$ model. Instead, there is evidence that the rôle of $\mathrm{T}$ and $\mathrm{S}$ duality is exchanged for the 5 brane (which is dual to the string in $D=10$ ) and that string $\mathrm{S}$ duality can be discovered as a symmetry of the 5 brane worldvolume theory [16].

The situation is quite different for $M$ theory. Since, in this theory, the dilaton is just a geometrical modulus (associated with the radius of the compact eleventh dimension), one does not expect a conceptual difference between $\mathrm{S}$ and $\mathrm{T}$ duality. Consequently, one may hope to find both symmetries, and even the larger U-duality symmetry, from a single worldvolume theory; namely, the $\hat{D}=11$ supermembrane. Then, formally, $\mathrm{U}$ duality would arise from the supermembrane in the same way that $\mathrm{T}$ duality arises from the string worldsheet : either as a classical continuous symmetry rotating equations of motion and Bianchi identities into each other or as a discrete symmetry leaving the "spectrum" of the membrane invariant. For both pictures, there exists some evidence in the literature. In a very interesting paper [17], Duff and Lu analyzed the moduli part of the membrane equations of motion and showed explicitly the appearance of the $D=7 \mathrm{U}$-duality group $S L(5)$. The present paper is highly motivated by this work. U duality as a symmetry of the membrane spectrum has been discussed by Sen [18]. He pointed out that, unlike for the string, the dimension $d$ of the momentum vector (for a membrane compactified to $D=11-d$ dimensions) generally does not equal the dimension of the winding vector which is $\frac{d(d-1)}{2}$. For $D=8$ only both dimensions coincide, so that there is a close analogy to the string in this dimension. In this case, Sen argued for the existence of an $S L(2)$ symmetry which leaves the theory invariant by exchanging momentum and winding numbers of the membrane and simultaneously transforming the dilaton and the 3 form modulus.

In this paper, we will analyze $\mathrm{U}$ duality rotations from the viewpoint of the classical membrane worldvolume theory, following the method of ref. [17. Our work differs from ref. 17] in two important aspects. First, we are considering the full 11-dimensional membrane target space, which appears to be crucial for the interpretation of the worldvolume symmetries as U-duality symmetries. Second, we include the full spectrum of bosonic background fields in our analysis. This means that we are looking for a continuous version of Buscher duality for the membrane. The outline of the paper is as follows. In the next section, we present a review of $\mathrm{T}$ duality rotations on the string worldsheet to explain the methods which we will apply to the membrane later on. Section 3 presents the general analysis for the membrane which follows the string analog as closely as possible. In section 4, we concentrate on pure moduli background field configurations and discuss all cases with 
$D \geq 6$. In particular, we reproduce the $D=7$ result of Duff and Lu and show how the $S L(2)$ symmetry of Sen arises within our setting. The general case, including all background field, is discussed in section 5 for $D=8$. Although we find that the $S L(2)$ part of the $D=8 \mathrm{U}$-duality group is obstructed as a worldvolume symmetry, we are able to read off the exact $S L(2) \times S L(3)$ transformation laws for all fields except for the 3 form. These results are verified in section 6 by comparison with $\hat{D}=11$ supergravity dimensionally reduced to $D=8$. Finally, in section 7 we summarize and comment on our results.

\section{Review of T Duality Rotations on the String Worldsheet}

In this section we will discuss how $\mathrm{T}$ duality rotations of a toroidally compactified string arise as symmetries of the worldsheet $\sigma$-model. The basic method which we will use is the analysis of rotations between equations of motion and Bianchi identities as first discussed by Gaillard and Zumino [10] in the context of 4-dimensional gauge theories. This method has been applied to the moduli part of the string worldsheet action by Duff [9] and we will follow the method of this paper. The generalization to the full background field content has been provided by Maharana and Schwarz [11]. Our main intention is to explain some of the methods, which we will later on apply to the membrane worldvolume theory, within the more familiar setting of string theory. Consequently, we will keep our presentation as close as possible to the membrane case. In particular, unlike in ref. [11, we will not work in conformal gauge but keep a general worldsheet metric. Furthermore, since we wish to discuss the full content of background fields for the membrane, we will do so for the string as well.

We consider a string in $\hat{D}=10$ dimensions with worldsheet coordinates $\xi^{i}, i, j, k, \ldots=0,1$, worldsheet metric $\gamma_{i j}$ and target space coordinates $X^{M}\left(\xi^{i}\right)$. The full target space is indexed by uppercase letters $M, N, P, \ldots=0, \ldots, \hat{D}-1$. Its motion in the background specified by the metric $\hat{g}_{M N}=\hat{g}_{M N}\left(X^{P}\right)$ and the antisymmetric tensor field $\hat{b}_{M N}=\hat{b}_{M N}\left(X^{P}\right)$ is described by the $\sigma-$ model Lagrangian 円

$$
\mathcal{L}=\frac{1}{2} \sqrt{-\gamma} \gamma^{i j} \partial_{i} X^{M} \partial_{j} X^{N} \hat{g}_{M N}+\frac{1}{2} \epsilon^{i j} \partial_{i} X^{M} \partial_{j} X^{N} \hat{b}_{M N}
$$

The equation of motion for $\gamma^{i j}$ implies the vanishing of the energy momentum $T_{i j}$ tensor and reads

$$
T_{i j}=\frac{1}{\sqrt{-\gamma}} \frac{\partial \mathcal{L}}{\partial \gamma^{i j}}=\frac{1}{2}\left(\partial_{i} X^{M} \partial_{j} X^{N} \hat{g}_{M N}-\gamma_{i j} \gamma^{k l} \partial_{k} X^{M} \partial_{l} X^{N} \hat{g}_{M N}\right)=0 .
$$

Next we would like to dimensionally reduce the above Lagrangian to $D=\hat{D}-d$ dimensions. We split the target space coordinates into an external and internal piece as $X^{M}=\left(X^{\mu}, X^{m}\right)$ and assume that the background depends on the external coordinates only; that is, $\hat{g}_{M N}=\hat{g}_{M N}\left(X^{\mu}\right)$ and $\hat{b}_{M N}=\hat{b}_{M N}\left(X^{\mu}\right)$. Indices $\mu, \nu, \rho, \ldots=0, \ldots, D-1$ and $m, n, r, \ldots=D, \ldots, \hat{D}-1$ are used

\footnotetext{
${ }^{1}$ Our definition of the $\epsilon$ symbol is such that $\epsilon^{01}=1$.
} 
to denote the external and internal coordinates respectively. The background fields are split in a corresponding way as $\hat{g}_{M N}=\left(\hat{g}_{\mu \nu}, \hat{g}_{\mu n}, \hat{g}_{m n}\right)$ and $\hat{b}_{M N}=\left(\hat{b}_{\mu \nu}, \hat{b}_{\mu n}, \hat{b}_{m n}\right)$ where $\hat{g}_{\mu \nu}, \hat{b}_{\mu \nu}$ are the external metric and antisymmetric tensor field, $\hat{g}_{\mu n}, \hat{b}_{\mu n}$ represent $2 d$ vector fields on the external space and $\hat{g}_{m n}, \hat{b}_{m n}$ are the $d^{2}$ moduli of the internal space. The Lagrangian (1) then decomposes into an external part, a mixed part and an internal, pure moduli part as

$$
\mathcal{L}=\mathcal{L}^{(0)}+\mathcal{L}^{(1)}+\mathcal{L}^{(2)}
$$

with

$$
\begin{aligned}
\mathcal{L}^{(0)} & =\frac{1}{2} \sqrt{-\gamma} \gamma^{i j} \partial_{i} X^{\mu} \partial_{j} X^{\nu} \hat{g}_{\mu \nu}+\frac{1}{2} \epsilon^{i j} \partial_{i} X^{\mu} \partial_{j} X^{\nu} \hat{b}_{\mu \nu} \\
\mathcal{L}^{(1)} & =\sqrt{-\gamma} \gamma^{i j} \partial_{i} X^{\mu} \partial_{j} X^{n} \hat{g}_{\mu n}+\epsilon^{i j} \partial_{i} X^{\mu} \partial_{j} X^{n} \hat{b}_{\mu n} \\
\mathcal{L}^{(2)} & =\frac{1}{2} \sqrt{-\gamma} \gamma^{i j} \partial_{i} X^{m} \partial_{j} X^{n} \hat{g}_{m n}+\frac{1}{2} \epsilon^{i j} \partial_{i} X^{m} \partial_{j} X^{n} \hat{b}_{m n} .
\end{aligned}
$$

Since $\mathcal{L}$ depends on the coordinates $X^{m}$ through their derivatives only, we may introduce "field strengths" $F_{i}^{m}$ and rewrite $\mathcal{L}$ in an equivalent first order form as

$$
\mathcal{L}_{x}=\left.\mathcal{L}^{(0)}\right|_{\partial X=F}-\left.\mathcal{L}^{(2)}\right|_{\partial X=F}+\left.\frac{\partial \mathcal{L}}{\partial\left(\partial_{i} X^{m}\right)}\right|_{\partial X=F} \partial_{i} X^{m}
$$

where $\left.\right|_{\partial X=F}$ after an expression indicates that $\partial_{i} X^{m}$ has been replaced by $F_{i}^{m}$. The equivalence of this first order Lagrangian to the original one can be easily proven by looking at the equation of motion for $F_{i}^{m}$,

$$
\frac{\partial \mathcal{L}_{x}}{\partial F_{i}^{m}}=\left(\sqrt{-\gamma} \gamma^{i j} \hat{g}_{m n}+\epsilon^{i j} \hat{b}_{m n}\right)\left(\partial_{i} X^{m}-F_{i}^{m}\right)=0
$$

which leads to $F_{i}^{m}=\partial_{i} X^{m}$. Substituting this solution into $\mathcal{L}_{x}$ leads back to $\mathcal{L}$. That is

$$
\mathcal{L}=\left.\mathcal{L}_{x}\right|_{F=\partial X}
$$

Consequently, the equations of motion for $\mathcal{L}$ and $\mathcal{L}_{x}$ are completely equivalent. In particular, the conjugate momenta of $X^{M}$ are related to each other by exchange of $F_{i}^{m}$ and $\partial_{i} X^{m}$

$$
\frac{\partial \mathcal{L}_{x}}{\partial\left(\partial_{i} X^{M}\right)}=\left.\frac{\partial \mathcal{L}}{\partial\left(\partial_{i} X^{M}\right)}\right|_{\partial X=F}
$$

with their internal and external components explicitly given by

$$
\begin{aligned}
& \left.\frac{\partial \mathcal{L}_{x}}{\partial\left(\partial_{i} X^{m}\right)}\right|_{F=\partial X}=\sqrt{-\gamma} \gamma^{i j} \partial_{j} X^{n} \hat{g}_{m n}+\epsilon^{i j} \partial_{j} X^{n} \hat{b}_{m n}+\sqrt{-\gamma} \gamma^{i j} \partial_{j} X^{\nu} \hat{g}_{m \nu}+\epsilon^{i j} \partial_{j} X^{\nu} \hat{b}_{m \nu} \\
& \left.\frac{\partial \mathcal{L}_{x}}{\partial\left(\partial_{i} X^{\mu}\right)}\right|_{F=\partial X}=\sqrt{-\gamma} \gamma^{i j} \partial_{j} X^{n} \hat{g}_{\mu n}+\epsilon^{i j} \partial_{j} X^{n} \hat{b}_{\mu n}+\sqrt{-\gamma} \gamma^{i j} \partial_{j} X^{\nu} \hat{g}_{\mu \nu}+\epsilon^{i j} \partial_{j} X^{\nu} \hat{b}_{\mu \nu} .
\end{aligned}
$$

Similarly, the energy momentum tensor $T_{i j}^{(x)}$ of $\mathcal{L}_{x}$ can be expressed as

$$
T_{i j}^{(x)} \equiv \frac{1}{\sqrt{-\gamma}} \frac{\partial \mathcal{L}_{x}}{\partial \gamma^{i j}}=\left.\left.\frac{1}{\sqrt{-\gamma}} \frac{\partial \mathcal{L}}{\partial \gamma^{i j}}\right|_{\partial X=F} \equiv T_{i j}\right|_{\partial X=F}
$$


We now observe that the equation of motion and the Bianchi identity for $X^{m}$ can be written in the following form

$$
\partial_{i}\left(\begin{array}{c}
\frac{\partial \mathcal{L}}{\partial\left(\partial_{i} X^{m}\right)} \\
\epsilon^{i j} \partial_{j} X^{m}
\end{array}\right)=0
$$

where the conjugate momenta are given by eq. (9). This suggests the existence of a duality symmetry rotating conjugate momenta and the dual field strengths $\epsilon^{i j} \partial_{j} X^{m}$ of the internal target space coordinates into each other. Such a symmetry should leave the other equations of motion (the ones for $X^{\mu}$ ) invariant. Later, we will see that already the external conjugate momenta (10) are invariant by themselves.

Equation (12) shows an apparent asymmetry between its upper and lower part and is clearly not written in a manifest duality invariant form. To find such a form it is useful to introduce a dual Lagrangian $\mathcal{L}_{y}$ with the rôle of equations of motion and Bianchi identities being exchanged. We dualize the internal coordinates $X^{m}$ to coordinates $Y_{m}$ by defining

$$
\mathcal{L}_{y}=\left.\mathcal{L}\right|_{\partial X=F}+\epsilon^{i j} \partial_{i} Y_{m} F_{j}^{m}
$$

Then the conjugate momentum for $Y_{m}$ is given by

$$
\frac{\partial \mathcal{L}_{y}}{\partial\left(\partial_{i} Y_{m}\right)}=\epsilon^{i j} F_{j}^{m}
$$

This leads to the equation of motion $\epsilon^{i j} \partial_{i} F_{j}^{m}=0$ which implies that $F_{i}^{m}=\partial_{i} X^{m}$ locally. Furthermore, from the definition of $\mathcal{L}_{y}$ and the eqs. (7), (8), (11) we have the following relations

$$
\begin{aligned}
\frac{\partial \mathcal{L}_{y}}{\partial F_{i}^{m}} & =\frac{\partial \mathcal{L}_{x}}{\partial \partial_{i} X^{m}}-\epsilon^{i j} \partial_{j} Y_{m}=0 \\
\frac{\partial \mathcal{L}_{y}}{\partial\left(\partial_{i} X^{\mu}\right)} & =\frac{\partial \mathcal{L}_{x}}{\partial\left(\partial_{i} X^{\mu}\right)} \\
\frac{\partial \mathcal{L}_{y}}{\partial X^{\mu}} & =\frac{\partial \mathcal{L}_{x}}{\partial X^{\mu}} \\
T_{i j}^{(y)} \equiv \frac{1}{\sqrt{-\gamma}} \frac{\partial \mathcal{L}_{y}}{\partial \gamma^{i j}} & =T_{i j}^{(x)}
\end{aligned}
$$

Taking the derivative $\partial_{i}$ of the first of these equations shows that the $\mathcal{L}_{y}$ equation of motion for $F_{i}^{m}$ implies the $\mathcal{L}_{x}$ equation of motion for $X^{m}$. The second and third equation show that $\mathcal{L}_{y}$ and $\mathcal{L}_{x}$ lead to the same equations of motion for $X^{\mu}$ and, finally, the fourth equation shows the equality of the energy momentum tensors. Therefore, the theories defined by $\mathcal{L}_{x}$ and $\mathcal{L}_{y}$ are classically equivalent.

We are now able to pair the internal conjugate momenta and the dual field strengths in a more symmetric way. Using the eqs. (15) and (14) along with the internal conjugate momentum of $\mathcal{L}_{x}$ 
in eq. (9) and $F_{i}^{m}=\partial_{i} X^{m}$ we find

$$
\begin{aligned}
\epsilon^{i j} \partial_{j} Y_{m}= & \frac{\partial \mathcal{L}_{x}}{\partial\left(\partial_{i} X^{m}\right)}= \\
& \hat{g}_{m n} \sqrt{-\gamma} \gamma^{i j} \partial_{j} X^{n}+\hat{b}_{m n} \epsilon^{i j} \partial_{j} X^{n}+\hat{g}_{m \nu} \sqrt{-\gamma} \gamma^{i j} \partial_{j} X^{\nu} \\
& +\hat{b}_{m \nu} \epsilon^{i j} \partial_{j} X^{\nu} \\
\epsilon^{i j} \partial_{j} X^{m}= & \frac{\partial \mathcal{L}_{y}}{\partial\left(\partial_{i} Y_{m}\right)}
\end{aligned}
$$

This shows that the internal conjugate momentum of $\mathcal{L}_{x}$ equals the dual field strength of $\mathcal{L}_{y}$ and vice versa. As a final step we would like to find an explicit expression for the $\mathcal{L}_{y}$ conjugate momentum which is still missing on the right hand side of the second equation above. This can be done by solving for $F_{i}^{m}=\partial_{i} X^{m}$ in terms of $Y_{m}$ by using the equation of motion (15) for $F_{i}^{m}$. Explicitly, this equation reads

$$
\sqrt{-\gamma} \gamma^{i j} F_{j}^{n} \hat{g}_{m n}+\epsilon^{i j} F_{j}^{n} \hat{b}_{m n}+\sqrt{-\gamma} \gamma^{i j} \partial_{j} X^{\nu} \hat{g}_{m \nu}+\epsilon^{i j} \partial_{j} X^{\nu} \hat{b}_{m \nu}-\epsilon^{i j} \partial_{j} Y_{m}=0
$$

Its solution is given by

$$
F_{i}^{m}=\widetilde{\hat{g}}^{m n} \frac{1}{\sqrt{-\gamma}} \epsilon_{i}{ }^{j} \partial_{j} Y_{n}+\widetilde{\widehat{b}}^{m n} \partial_{i} Y_{n}+\widetilde{\hat{g}}_{\nu}^{m} \frac{1}{\sqrt{-\gamma}} \epsilon_{i}^{j} \partial_{j} X^{\nu}+\widetilde{\widehat{b}}_{\nu}^{m} \partial_{i} X^{\nu}
$$

where the dual background fields are defined by

$$
\begin{aligned}
\widetilde{\hat{g}}_{m n} & =\hat{g}_{m n}+\hat{b}_{m r} \hat{g}^{r s} \hat{b}_{n s} \\
\widetilde{\hat{b}}^{m n} & =-\widetilde{\widehat{g}}^{m r} \hat{b}_{r s} \hat{g}^{s n} \\
\widetilde{\widehat{g}}_{\nu}^{m} & =-\widetilde{\widetilde{g}}^{m r} \hat{b}_{r \nu}-\widetilde{\hat{b}}^{m r} \hat{g}_{r \nu} \\
\widetilde{\hat{b}}_{\nu}^{m} & =-\widetilde{\widehat{b}}^{m r} \hat{b}_{r \nu}-\widetilde{\widehat{g}}^{m r} \hat{g}_{r \nu} .
\end{aligned}
$$

Inserting this result into eq. (20) leads to the explicit expression for the conjugate momentum of $Y_{m}$. Then (19) and (20) take the form

$$
\begin{aligned}
\epsilon^{i j} \partial_{j} Y_{m}=\frac{\partial \mathcal{L}_{x}}{\partial \partial_{i} X^{m}}= & \hat{g}_{m n} \sqrt{-\gamma} \gamma^{i j} \partial_{j} X^{n}+\hat{b}_{m n} \epsilon^{i j} \partial_{j} X^{n} \\
& +\hat{g}_{m \nu} \sqrt{-\gamma} \gamma^{i j} \partial_{j} X^{\nu}+\hat{b}_{m \nu} \epsilon^{i j} \partial_{j} X^{\nu} \\
\epsilon^{i j} \partial_{j} X^{m}=\frac{\partial \mathcal{L}_{y}}{\partial\left(\partial_{i} Y_{m}\right)}= & \widehat{\widetilde{g}}^{m n} \sqrt{-\gamma} \gamma^{i j} \partial_{j} Y_{n}+\widetilde{\widehat{b}}^{m n} \epsilon^{i j} \partial_{j} Y_{n} \\
& +\widetilde{\hat{g}}^{m}{ }_{\nu} \sqrt{-\gamma} \gamma^{i j} \partial_{j} X^{\nu}+\widetilde{\widehat{b}}^{m}{ }_{\nu} \epsilon^{i j} \partial_{j} X^{\nu} .
\end{aligned}
$$

These two equations are identical in form and can be obtained from each other by an exchange of $X^{m}$ with $Y_{m}$ and the background fields with their duals as defined in eq. (23). This result could be expected, and reflects the well known fact that the dual Lagrangian $\mathcal{L}_{y}$ expressed in terms of $Y_{m}$ (computed from eq. (13) by integrating out $F_{i}^{m}$ via eq. (22)) is of the same form as $\mathcal{L}$. As we will see this is no longer true in the case of the membrane. 
In order to read off the symmetry from eqs. (24) and (25) we solve for the vertex operators $\epsilon^{i j} \partial_{j} Y_{m}$ and $\epsilon^{i j} \partial_{j} X^{m}$ which still appear on the right hand side. This leads to

$$
\begin{aligned}
\left(\begin{array}{c}
\epsilon^{i j} \partial_{j} Y_{m} \\
\epsilon^{i j} \partial_{j} X^{m}
\end{array}\right)= & \left(\begin{array}{cc}
\widetilde{\hat{g}}_{m n} & \hat{b}_{m r} \hat{g}^{n r} \\
\hat{g}^{m r} \hat{b}_{n r} & \hat{g}^{m n}
\end{array}\right)\left[\left(\begin{array}{c}
\sqrt{-\gamma} \gamma^{i j} \partial_{j} X^{n} \\
\sqrt{-\gamma} \gamma^{i j} \partial_{j} Y_{n}
\end{array}\right)+\left(\begin{array}{c}
\hat{g}^{n r} \hat{g}_{r \nu} \\
\widetilde{\hat{g}}_{n r} \widetilde{\widehat{g}}_{\nu}^{r}
\end{array}\right) \sqrt{-\gamma} \gamma^{i j} \partial_{j} X^{\nu}\right. \\
& \left.+\left(\begin{array}{c}
\hat{g}^{n r} \hat{b}_{r \nu} \\
\widetilde{\hat{g}}_{n r} \widehat{\widetilde{b}}_{\nu}^{r}
\end{array}\right) \epsilon^{i j} \partial_{j} X^{\nu}\right]
\end{aligned}
$$

Let us introduce the following abbreviations

$$
\begin{aligned}
G_{m n} & =\hat{g}_{m n} \\
B_{m n} & =\hat{b}_{m n} \\
A_{\mu}^{(1) n} & =\hat{g}^{n r} \hat{g}_{\mu r} \\
A_{\mu n}^{(2)} & =\widetilde{\hat{g}}_{n r} \widetilde{\hat{g}}_{\mu}^{r}=\hat{b}_{\mu n}+B_{n r} A_{\mu}^{(1) r} \\
V_{\mu}^{(1) n} & =\hat{g}^{n r} \hat{b}_{r \mu} \\
V_{\mu n}^{(2)} & =\widetilde{\widehat{g}}_{n r} \widetilde{\hat{b}}_{\mu}^{r}=-\hat{g}_{\mu n}+B_{n r} V_{\mu}^{(1) r}
\end{aligned}
$$

Furthermore, to write eq. (26) in a more compact form we introduce quantities in the $2 d$-dimensional vector space spanned by the internal coordinates $X^{m}$ and their duals $Y_{m}$. First we define the $2 d \times 2 d$ matrix (using matrix notation for $G_{m n}$ and $B_{m n}$ )

$$
M=\left(\begin{array}{cc}
G-B^{T} G^{-1} B & B G^{-1} \\
-G^{-1} B & G^{-1}
\end{array}\right),
$$

which contains the metric and antisymmetric tensor moduli and serves as a parameterization of the moduli space. The two vectors

$$
\mathbf{A}_{\nu}=\left(\begin{array}{c}
A_{\nu}^{(1) n} \\
A_{\nu n}^{(2)}
\end{array}\right), \quad \mathbf{V}_{\nu}=\left(\begin{array}{l}
V_{\nu}^{(1) n} \\
V_{\nu n}^{(2)}
\end{array}\right)
$$

contain the vector fields of the theory; that is, the graviphotons and the ones arising form the antisymmetric tensor. Finally, we use the following short hand notation for the vertex operators

$$
\begin{gathered}
\widetilde{\mathcal{F}}=\left(\begin{array}{c}
\epsilon^{i j} \partial_{j} X^{m} \\
\epsilon^{i j} \partial_{j} Y_{m}
\end{array}\right), \quad \mathcal{F}=\left(\begin{array}{c}
\sqrt{-\gamma} \gamma^{i j} \partial_{j} X^{n} \\
\sqrt{-\gamma} \gamma^{i j} \partial_{j} Y_{n}
\end{array}\right) \\
\mathcal{A}^{\nu}=\sqrt{-\gamma} \gamma^{i j} \partial_{j} X^{\nu}, \quad \mathcal{V}^{\nu}=\epsilon^{i j} \partial_{j} X^{\nu}
\end{gathered}
$$

Note, that $\mathcal{F}$ and $\widetilde{\mathcal{F}}$ are vectors on the $\left(X^{m}, Y_{m}\right)$ space whereas $\mathcal{A}^{\nu}$ and $\mathcal{V}^{\nu}$ are scalars. Since the worldvolume index $i$ appears as an overall index in what follows it has been suppressed in this notation. With these definitions, eq. (25) can be written as

$$
\eta \widetilde{\mathcal{F}}=M\left(\mathcal{F}+\mathbf{A}_{\nu} \mathcal{A}^{\nu}+\mathbf{V}_{\nu} \mathcal{V}^{\nu}\right)
$$


with $\eta$ given by

$$
\eta=\left(\begin{array}{ll}
0 & \mathbf{1}_{d} \\
\mathbf{1}_{d} & 0
\end{array}\right) .
$$

Using this form of the internal equations of motion, we are now in a position to discuss duality rotations. We start by performing the following transformation on the vertex operators

$$
\begin{aligned}
\widetilde{\mathcal{F}} \rightarrow P^{-1^{T}} \widetilde{\mathcal{F}}, & \mathcal{F} \rightarrow P^{-1^{T} \mathcal{F}} \\
\mathcal{A}^{\nu} \rightarrow \mathcal{A}^{\nu}, & \mathcal{V}^{\nu} \rightarrow \mathcal{V}^{\nu}
\end{aligned}
$$

where $P$ is an invertible $2 d \times 2 d$ matrix. Note, that we have not transformed the external vertex operators $\mathcal{A}^{\nu}$ and $\mathcal{V}^{\nu}$, as suggested by their index structure. In order to keep the form of eq. (32) invariant under this transformation we should counter rotate the background as

$$
M \rightarrow P M P^{T}, \quad \mathbf{A}_{\nu} \rightarrow P^{-1^{T}} \mathbf{A}_{\nu}, \quad \mathbf{V}_{\nu} \rightarrow P^{-1^{T}} \mathbf{V}_{\nu}
$$

In general, the transformation law for $M$ will not preserve its structure so that we have to restrict the set of allowed matrices $P$. We notice that, from its definition (28), $M$ is symmetric and fulfills the equation $M \eta M=\eta$. The latter property means that $M$ is an element of $O(d, d)$. In fact, $M$ is a parameterization of the moduli coset $O(d, d) / O(d) \times O(d)$. Therefore, the group of duality rotations which leaves eq. (32) invariant is given by $O(d, d)$ so that $P$ is constrained by

$$
P^{T} \eta P=\eta
$$

The quantities $\mathbf{A}_{\nu}$ and $\mathbf{V}_{\nu}$ contain the same degrees of freedom, namely the $2 d$ vector fields of the reduced theory. One might therefore ask, whether the transformations assigned to them in eq. (34) are compatible with each other. From the definitions of $\mathbf{A}_{\nu}$ and $\mathbf{V}_{\nu}$, eq. (29), and the definition of $M$, eq. (28), we deduce

$$
\mathbf{V}_{\nu}=-\eta M \mathbf{A}_{\nu}
$$

which is indeed consistent with the transformations (34) using eq. (35).

So far, we have assigned $O(d, d)$ transformation properties to the moduli in $M$ and the vector fields in $\mathbf{A}_{\nu}$ and $\mathbf{V}_{\nu}$. Clearly, we would also like obtain the $O(d, d)$ properties of the external metric $\hat{g}_{\mu \nu}$ and the external antisymmetric tensor $\hat{b}_{\mu \nu}$. They can be read off from the external equations of motion for $X^{\mu}$, once these are brought into a manifest $O(d, d)$ invariant form. In conformal gauge, this has been done in ref. [11]. Here, we will not present the invariant form of the full equations of motion but concentrate on the conjugate momenta for $X^{\mu}$. Using the expression (10) for these conjugate momenta, and inserting the eqs. (26) repeatedly, we arrive at

$$
\frac{\partial \mathcal{L}_{x}}{\partial\left(\partial_{i} X^{\mu}\right)}=\bar{g}_{\mu \nu} \mathcal{A}^{i \nu}+\left(B_{\mu \nu}+\frac{1}{2} \mathbf{A}_{\mu}^{T} \eta \mathbf{A}_{\nu}\right) \mathcal{V}^{i \nu}+\mathbf{A}_{\mu}^{T} \eta \widetilde{\mathcal{F}}^{i}
$$


with

$$
\begin{aligned}
\bar{g}_{\mu \nu} & =\hat{g}_{\mu \nu}-A_{\mu}^{(1) n} A_{\nu n}^{(1)} \\
B_{\mu \nu} & =\hat{b}_{\mu \nu}+\frac{1}{2} A_{\mu}^{(1) r} A_{\nu r}^{(2)}-\frac{1}{2} A_{\nu}^{(1) r} A_{\mu r}^{(2)}-B_{r s} A_{\mu}^{(1) r} A_{\nu}^{(1) s} .
\end{aligned}
$$

Given the known transformations (34), we conclude that the external conjugate momenta are invariant if $\bar{g}_{\mu \nu}$ and $B_{\mu \nu}$ as defined above are $O(d, d)$ singlets, that is

$$
\bar{g}_{\mu \nu} \rightarrow \bar{g}_{\mu \nu}, \quad B_{\mu \nu} \rightarrow B_{\mu \nu}
$$

To summarize, for a $\hat{D}$-dimensional string $\sigma$-model dimensionally reduced to $D=\hat{D}-d$ dimensions, we have demonstrated the existence of an $O(d, d)$ symmetry which leaves the equations of motion invariant. This symmetry acts on the vertex operators as in eq. (33) and on the background fields as in eq. (34), (40). An independent check for the background transformation laws is provided by the effective low energy action. Its dimensional reduction to $D$ dimensions should lead to a theory which is invariant under the $O(d, d)$ symmetry, acting on the background fields. In particular, the reduced effective action should be expressible in terms of invariant combinations of the $O(d, d)$ covariant quantities $M, \mathbf{A}_{\nu}, \bar{g}_{\mu \nu}$ and $B_{\mu \nu}$. That this is indeed true has been shown in ref. 11]. For the membrane, we will use this observation to confirm the background transformations obtained from the worldvolume theory by dimensional reduction of $\hat{D}=11$ supergravity.

\section{Duality on the Membrane Worldvolume}

Now we would like to apply the method of the previous section to the bosonic part of the $\hat{D}=11$ supermembrane [3, 4, 15]. For the moduli part this has first been done by Duff and Lu [17]. Here we will keep the full background field content of the theory.

We denote the three worldvolume coordinates of the membrane by $\xi^{i}, i, j, k, \ldots=0,1,2$ and the worldvolume metric with signature $(-++)$ by $\gamma_{i j}$. The target space coordinates $X^{M}\left(\xi^{i}\right)$ are indexed by uppercase letter $M, N, P, \ldots=0, \ldots, 10$. The bosonic background field content of the supermembrane is given by a metric $\hat{g}_{M N}=\hat{g}_{M N}\left(X^{R}\right)$ and a 3 -form field $\hat{b}_{M N P}=\hat{b}_{M N P}\left(X^{R}\right)$. With these definitions, the bosonic part of the supermembrane Lagrangian reads

$$
\mathcal{L}=\frac{1}{2} \sqrt{-\gamma} \gamma^{i j} \partial_{i} X^{M} \partial_{j} X^{N} \hat{g}_{M N}+\frac{1}{6} \epsilon^{i j k} \partial_{i} X^{M} \partial_{j} X^{N} \partial_{k} X^{P} \hat{b}_{M N P}-\frac{1}{2} \sqrt{-\gamma} .
$$

Note that this Lagrangian contains a cosmological constant term. For the string $\sigma$-model such a term was forbidden by conformal invariance. This leads to a modified expression for the energy momentum tensor

$$
T_{i j} \equiv \frac{1}{\sqrt{-\gamma}} \frac{\partial \mathcal{L}}{\partial \gamma^{i j}}=\frac{1}{2}\left(\partial_{i} X^{M} \partial_{j} X^{N} \hat{g}_{M N}-\gamma_{i j} \gamma^{k l} \partial_{k} X^{M} \partial_{l} X^{N} \hat{g}_{M N}+\frac{1}{2} \gamma_{i j}\right)=0 .
$$


The vanishing of $T_{i j}$ now implies that $\gamma_{i j}$ is the induced metric on the worldvolume

$$
\gamma_{i j}=\partial_{i} X^{M} \partial_{j} X^{N} \hat{g}_{M N}
$$

For the dimensional reduction of the Lagrangian (41) to $D=\hat{D}-d$ dimensions we split the target space coordinates as $X^{M}=\left(X^{\mu}, X^{m}\right)$. The external coordinates $X^{\mu}$ are indexed by $\mu, \nu, \rho, \ldots=$ $0, \ldots, D-1$ and the internal coordinates $X^{m}$ by $m, n, r, \ldots=D, \ldots, \hat{D}-1$. We assume that the background fields are independent on the internal coordinates $X^{m}$; that is, $\hat{g}_{M N}=\hat{g}_{M N}\left(X^{\mu}\right)$ and $\hat{b}_{M N R}=\hat{b}_{M N R}\left(X^{\mu}\right)$. Under this split of coordinates, the background fields break up as $\hat{g}_{M N}=\left(\hat{g}_{\mu \nu}, \hat{g}_{\mu n}, \hat{g}_{m n}\right), \hat{b}_{M N R}=\left(\hat{b}_{\mu \nu \rho}, \hat{b}_{\mu \nu r}, \hat{b}_{\mu n r}, \hat{b}_{m n r}\right)$. The background field content of the reduced theory is therefore given by the metric $\hat{g}_{\mu \nu}$ and the 3 -form $\hat{b}_{\mu \nu \rho}$, the $d 2$-forms $\hat{b}_{\mu \nu r}$, the $d(d+1) / 2$ vector fields $\hat{g}_{\mu n}, \hat{b}_{\mu n r}$ and the $d\left(d^{2}+5\right) / 6$ moduli fields $\hat{g}_{m n}, \hat{b}_{m n r}$. The Lagrangian (41) can be decomposed as

$$
\mathcal{L}=\sum_{n=0}^{3} \mathcal{L}^{(n)}
$$

with $\mathcal{L}^{(n)}$ being homogeneous of degree $n$ in the internal coordinates $X^{m}$. Explicitly, these various parts are given by

$$
\begin{aligned}
\mathcal{L}^{(0)} & =\frac{1}{2} \sqrt{-\gamma} \gamma^{i j} \partial_{i} X^{\mu} \partial_{j} X^{\nu} \hat{g}_{\mu \nu}+\frac{1}{6} \epsilon^{i j k} \partial_{i} X^{\mu} \partial_{j} X^{\nu} \partial_{k} X^{\rho} \hat{b}_{\mu \nu \rho}-\frac{1}{2} \sqrt{-\gamma} \\
\mathcal{L}^{(1)} & =\sqrt{-\gamma} \gamma^{i j} \partial_{i} X^{\mu} \partial_{j} X^{n} \hat{g}_{\mu n}+\frac{1}{2} \epsilon^{i j k} \partial_{i} X^{\mu} \partial_{j} X^{\nu} \partial_{k} X^{r} \hat{b}_{\mu \nu r} \\
\mathcal{L}^{(2)} & =\frac{1}{2} \sqrt{-\gamma} \gamma^{i j} \partial_{i} X^{m} \partial_{j} X^{n} \hat{g}_{m n}+\frac{1}{2} \epsilon^{i j k} \partial_{i} X^{\mu} \partial_{j} X^{n} \partial_{k} X^{r} \hat{b}_{\mu n r} \\
\mathcal{L}^{(3)} & =\frac{1}{6} \epsilon^{i j k} \partial_{i} X^{m} \partial_{j} X^{n} \partial_{k} X^{r} \hat{b}_{m n r} .
\end{aligned}
$$

Since $\mathcal{L}$ does not depend on $X^{m}$ explicitly, we may introduce first order fields $F_{i}^{m}$ and rewrite the Lagrangian as

$$
\mathcal{L}_{x}=\left.\sum_{n=0}^{3}(1-n) \mathcal{L}^{(n)}\right|_{\partial X=F}+\left.\frac{\partial \mathcal{L}}{\partial\left(\partial_{i} X^{m}\right)}\right|_{\partial X=F} \partial_{i} X^{m},
$$

where $\left.\right|_{\partial X=F}$ indicates that $\partial_{i} X^{m}$ has been replaced by $F_{i}^{m}$ in the respective expression. As in the string case, we can prove the equivalence of $\mathcal{L}$ and $\mathcal{L}_{x}$ by analyzing the equation of motion

$$
\frac{\partial \mathcal{L}_{x}}{\partial F_{i}^{m}}=0
$$

for $F_{i}^{m}$. Its solution, $F_{i}^{m}=\partial_{i} X^{m}$, substituted into $\mathcal{L}_{x}$ results in

$$
\mathcal{L}=\left.\mathcal{L}_{x}\right|_{F=\partial X}
$$

In particular, we have the following relations between the conjugate momenta of $\mathcal{L}$ and $\mathcal{L}_{x}$

$$
\frac{\partial \mathcal{L}_{x}}{\partial\left(\partial_{i} X^{M}\right)}=\left.\frac{\partial \mathcal{L}}{\partial\left(\partial_{i} X^{M}\right)}\right|_{\partial X=F}
$$


and the energy momentum tensors of $\mathcal{L}$ and $\mathcal{L}_{x}$

$$
T_{i j}^{(x)} \equiv \frac{1}{\sqrt{-\gamma}} \frac{\partial \mathcal{L}_{x}}{\partial \gamma^{i j}}=\left.\left.\frac{1}{\sqrt{-\gamma}} \frac{\partial \mathcal{L}}{\partial \gamma^{i j}}\right|_{\partial X=F} \equiv T_{i j}\right|_{\partial X=F}
$$

The latter relation implies that $\gamma_{i j}$ is still given by the induced metric as in eq. (43), but with $\partial_{i} X^{m}$ replaced by $F_{i}^{m}$. That is,

$$
\gamma_{i j}=F_{i}^{m} F_{j}^{n} \hat{g}_{m n}+F_{i}^{m} \partial_{j} X^{\nu} \hat{g}_{m \nu}+\partial_{i} X^{\mu} F_{j}^{n} \hat{g}_{\mu n}+\partial_{i} X^{\mu} \partial_{j} X^{\nu} \hat{g}_{\mu \nu}
$$

We remark that in ref. [17] only the first term in eq. (51) was taken into account. However, even if all background fields except the moduli are turned off, the last term in eq. (51) is still nonvanishing. As we will see, the presence of these extra terms complicates the subsequent calculation considerably.

The explicit expressions for the conjugate momenta are given by

$$
\begin{aligned}
& \left.\frac{\partial \mathcal{L}_{x}}{\partial\left(\partial_{i} X^{m}\right)}\right|_{F=\partial X}=\sqrt{-\gamma} \gamma^{i j} \partial_{j} X^{N} \hat{g}_{m N}+\frac{1}{2} \epsilon^{i j k} \partial_{j} X^{N} \partial_{k} X^{R} \hat{b}_{m N R} \\
& \left.\frac{\partial \mathcal{L}_{x}}{\partial\left(\partial_{i} X^{\mu}\right)}\right|_{F=\partial X}=\sqrt{-\gamma} \gamma^{i j} \partial_{j} X^{N} \hat{g}_{\mu N}+\frac{1}{2} \epsilon^{i j k} \partial_{j} X^{N} \partial_{k} X^{R} \hat{b}_{\mu N R} .
\end{aligned}
$$

As for the string, we would now like to pair the equations of motion for $X^{m}$ with Bianchi identities. What are the vertex operators for these Bianchi identities in the case of the membrane? A natural generalization of the string winding operator is $\epsilon^{i j k} \partial_{j} X^{m} \partial_{k} X^{n}$, which leads to the conservation equation

$$
\partial_{i}\left(\begin{array}{c}
\frac{\partial \mathcal{L}}{\partial\left(\partial_{i} X^{m}\right)} \\
\epsilon^{i j k} \partial_{j} X^{m} \partial_{k} X^{n}
\end{array}\right)=0
$$

with the conjugate momentum given in eq. (52). Note that we have paired $d$ conserved momenta and $d(d-1) / 2$ conserved winding numbers in the above equation. Unlike for the string, these numbers are not equal except for a dimensional reduction to $D=8(d=3)$. This reflects the fact that there exist $d(d-1) / 2$ ways for the two spatial directions of the membrane to wrap around $d$ compact directions. That the pairing in eq. (54) is reasonable is also suggested by the result in ref. [16]. There it has been shown that an analogous pairing for the 5 brane leads to charges which transform correctly under the $S L(2)$ S-duality of string theory. Another important difference from the string case is the existence of an additional "mixed" Bianchi identities

$$
\partial_{i}\left(\epsilon^{i j k} \partial_{j} X^{m} \partial_{k} X^{\nu}\right)=0
$$

We interpret these conserved currents as corresponding to membrane states with only one spatial coordinate wrapped around a compact direction.

Again, we would like to find a symmetric form of eq. (54) which shows the inferred duality symmetry in a manifest way. To do this, we construct a dual Lagrangian with the rôle of equations 
of motion and Bianchi identities exchanged. Having noticed the presence of the two types of Bianchi identities in eq. (54) and (55), a natural definition for this dual Lagrangian $\mathcal{L}_{y}$ is

$$
\mathcal{L}_{y}=\left.\mathcal{L}\right|_{\partial X=F}+\epsilon^{i j k} \partial_{i} Y_{m n} F_{j}^{m} F_{k}^{n}+\epsilon^{i j k} \partial_{i} Y_{m \nu} F_{j}^{m} \partial_{k} X^{\nu}
$$

We have introduced two types of coordinates "dual" to $X^{m}$, namely $Y_{m n}$ and $Y_{m \nu}$. Therefore $\mathcal{L}_{y}$, with the auxiliary field $F_{i}^{m}$ being integrated out, will be different in structure from $\mathcal{L}_{x}$. In particular, the target space dimension of $\mathcal{L}_{y}$ does not coincide with the one of $\mathcal{L}_{x}$. This is to be contrasted to the string case where both Lagrangians were of the same form.

To establish a relation between $\mathcal{L}_{y}$ and $\mathcal{L}_{x}$ we consider the conjugate momenta

$$
\begin{aligned}
\frac{\partial \mathcal{L}_{y}}{\partial\left(\partial_{i} Y_{m n}\right)} & =\epsilon^{i j k} F_{j}^{m} F_{k}^{n} \\
\frac{\partial \mathcal{L}_{y}}{\partial\left(\partial_{i} Y_{m \nu}\right)} & =\epsilon^{i j k} F_{j}^{m} \partial_{k} X^{\nu} .
\end{aligned}
$$

and the corresponding equations of motion

$$
\begin{aligned}
\partial_{i}\left(\epsilon^{i j k} F_{j}^{m} F_{k}^{n}\right) & =0 \\
\partial_{i}\left(\epsilon^{i j k} F_{j}^{m} \partial_{k} X^{\nu}\right) & =0 .
\end{aligned}
$$

Certainly, these equations are solved by $F_{i}^{m}=\partial_{i} X^{m}$. Is this really the only solution of the system (59), (60)? Let us consider a specific example for $d=2$. We take $F_{i}^{1}=\left(\xi^{0} \xi^{1}, 0,0\right)$ and $F_{i}^{2}=(0,1,0)$ which fulfill $\partial_{i}\left(\epsilon^{i j k} F_{j}^{1} F_{k}^{2}\right)=0$; that is, the $Y_{m n}$ equation of motion (59). On the other hand $\epsilon^{i j k} \partial_{j} F_{k}^{1}=\left(0,0,-\xi^{0}\right)$, which implies that $F_{i}^{1}$ is not closed (as a 1 -form on the world volume). This shows that adding the first Lagrange multiplier term in eq. (56) only, as was done in refs. [17, does not guarantee $F_{i}^{m}=\partial_{i} X^{m}$. Since eq. (59) provides $d(d-1) / 2$ linear homogeneous equations for the $3 d$ quantities $\epsilon^{i j k} \partial_{j} F_{k}^{m}$, we expect similar examples up to $d=6$ at least. Certainly, the additional mixed Bianchi identities (60) eliminate some of these cases. For the above example in $d=2$ we have $\partial_{i}\left(\epsilon^{i j k} F_{j}^{1} \partial_{k} X^{\nu}\right)=-\xi^{0} \partial_{2} X^{\nu}$, so that it does not solve the full system of equations (59), (60) if only $\partial_{2} X^{\nu} \neq 0$ for one $\nu$. Such a condition, however, is not guaranteed and can be violated for membranes oriented transversally to the external space. Therefore, though being an "improvement" over just using the first condition (59), the system (59), (60) still does not force $F_{i}^{m}=\partial_{i} X^{m}$ in general. A way to unambiguously obtain such a solution, is to replace the two Lagrange multiplier terms in eq. (56) by $\epsilon^{i j k} A_{j m} F_{k}^{m}$ where $A_{j m}$ are $d$ worldvolume vector fields. Their equation of motion is given by $\epsilon^{i j k} \partial_{j} F_{k}^{m}=0$ which implies $F_{i}^{m}=\partial_{i} X^{m}$ locally. Such a method can, for example, be used to derive the type IIA 2D-brane action from the 11-dimensional supermembrane [19]. In our context, however, the worldvolume vectors $A_{i m}$, unlike the fields $Y_{m n}$, $Y_{m \nu}$, are not the appropriate degrees of freedom to describe winding modes of the membrane. Correspondingly, pairing the original Lagrangian $\mathcal{L}_{x}$ with $\mathcal{L}_{y}$ defined in such a way does not show any of the expected U-duality symmetry structure. Alternatively, the term $\epsilon^{i j k} A_{j m} F_{k}^{m}$ could be 
added to the definition (56) of $\mathcal{L}_{y}$. Though there is nothing wrong with this in principle, it is hard to see what the interpretation of the additional vector fields $A_{j m}$ could be.

For the moment, we will therefore accept the somewhat unfortunate situation that the theory described by $\mathcal{L}_{y}$ in eq. (56) seems to be more general than the original one and concentrate on those solutions of $\mathcal{L}_{y}$ for which $F_{i}^{m}=\partial_{i} X^{m}$. Then we have from eq. (56) and the eqs. (48), (49), (50)

$$
\begin{aligned}
\frac{\partial \mathcal{L}_{y}}{\partial F_{i}^{m}} & =\frac{\partial \mathcal{L}_{x}}{\partial \partial_{i} X^{m}}-2 \epsilon^{i j k} \partial_{j} Y_{m n} F_{i}^{n}-2 \epsilon^{i j k} \partial_{j} Y_{m \nu} \partial_{k} X^{\nu}=0 \\
\frac{\partial \mathcal{L}_{y}}{\partial\left(\partial_{i} X^{\mu}\right)} & =\frac{\partial \mathcal{L}_{x}}{\partial\left(\partial_{i} X^{\mu}\right)}-2 \epsilon^{i j k} \partial_{j} Y_{\mu n} F_{k}^{n} \\
\frac{\partial \mathcal{L}_{y}}{\partial X^{\mu}} & =\frac{\partial \mathcal{L}_{x}}{\partial X^{\mu}} \\
T_{i j}^{(y)} \equiv \frac{1}{\sqrt{-\gamma}} \frac{\partial \mathcal{L}_{y}}{\partial \gamma^{i j}} & =T_{i j}^{(x)} .
\end{aligned}
$$

The additional terms in eq. (61) and (62) vanish once we set $F_{i}^{m}=\partial_{i} X^{m}$ and take the derivative $\partial_{i}$. This shows the classical equivalence of $\mathcal{L}_{x}$ and $\mathcal{L}_{y}$ provided the solutions of $\mathcal{L}_{y}$ are restricted to those with $F_{i}^{m}=\partial_{i} X^{m}$. From now on we will assume this restriction and use $F_{i}^{m}$ and $\partial_{i} X^{m}$ interchangeably.

Let us now rewrite the internal conjugate momenta and the Bianchi identity from eq. (54) in a more symmetric way using the Lagrangian $\mathcal{L}_{y}$. Putting together eq. (61), the internal conjugate momentum (52), eq. (57) and $F_{i}^{m}=\partial_{i} X^{m}$ we find

$$
\begin{aligned}
2 \epsilon^{i j k} \partial_{j} Y_{m N} \partial_{k} X^{N}=\frac{\partial \mathcal{L}_{x}}{\partial\left(\partial_{i} X^{m}\right)}= & \hat{g}_{m n} \sqrt{-\gamma} \gamma^{i j} \partial_{j} X^{n}+\frac{1}{2} \hat{b}_{m n r} \epsilon^{i j k} \partial_{j} X^{n} \partial_{k} X^{r}+\hat{g}_{m \nu} \sqrt{-\gamma} \gamma^{i j} \partial_{j} X^{\nu} \\
& +\hat{b}_{m n \nu} \epsilon^{i j k} \partial_{j} X^{n} \partial_{k} X^{\nu}+\frac{1}{2} \hat{b}_{m \rho \nu} \epsilon^{i j k} \partial_{j} X^{\rho} \partial_{k} X^{\nu} \\
\epsilon^{i j k} \partial_{j} X^{m} \partial_{k} X^{n}= & \frac{\partial \mathcal{L}_{y}}{\partial\left(\partial_{i} Y_{m n}\right)} .
\end{aligned}
$$

By construction of the dual Lagrangian $\mathcal{L}_{y}$, the conjugate momentum of $Y_{m n}$ equals the "Bianchi identity" $\epsilon^{i j k} \partial_{j} X^{m} \partial_{k} X^{n}$ of $\mathcal{L}_{x}$. The conjugate momentum of $X^{m}$, on the other hand, equals the operator $2 \epsilon^{i j k} \partial_{j} Y_{m N} \partial_{k} X^{N}$ which we interpret as the Bianchi identity of $\mathcal{L}_{y}$. Its unconventional form in terms of $X^{N}$ results because we have used Lagrange multiplier terms in eq. (56) which are bilinear in $\partial X$. Moreover, according to eq. (60), the mixed Bianchi identity equals the conjugate momentum of $Y_{m \nu}$

$$
\epsilon^{i j k} \partial_{j} X^{m} \partial_{k} X^{\nu}=\frac{\partial \mathcal{L}_{y}}{\partial\left(\partial_{i} Y_{m \nu}\right)} .
$$

As in the string case, we now have to find explicit expressions for the right hand sides of eq. (66) and (67) in terms of the dual coordinates $Y_{m N}$. These expressions should be obtained by solving eq. (61), which explicitly reads

$$
\sqrt{-\gamma} \gamma^{i j} F_{j}^{n} \hat{g}_{m n}+\frac{1}{2} \epsilon^{i j k} F_{j}^{n} F_{k}^{r} \hat{b}_{m n r}+\sqrt{-\gamma} \gamma^{i j} \partial_{j} X^{\nu} \hat{g}_{m \nu}+\epsilon^{i j k} F_{j}^{n} \partial_{k} X^{\rho} \hat{b}_{m n \rho}
$$




$$
+\frac{1}{2} \epsilon^{i j k} \partial_{j} X^{\nu} \partial_{k} X^{\rho} \hat{b}_{m \nu \rho}-2 \epsilon^{i j k} \partial_{j} Y_{m n} F_{k}^{n}-2 \epsilon^{i j k} \partial_{j} Y_{m \nu} \partial_{k} X^{\nu}=0
$$

Unfortunately, this equation cannot be simply solved for $F_{i}^{m}$ in terms of $Y_{m N}$ as in the string case because of the terms quadratic in $F_{i}^{m}$ and the appearance of $F_{i}^{m}$ in the second to last term (compare with eq. (21) for the string). The best we can do at this point is to either solve eq. (68) iteratively or to find an implicit solution. Unlike in the string case, it is therefore hard to find a closed form for the dual Lagrangian $\mathcal{L}_{y}$ with the auxiliary field $F_{i}^{m}$ being integrated out. Consequently, our main focus is on the equations of motion from which we attempt to read off the duality symmetry. To be able to do so, we are clearly interested in a closed form of these equations. Therefore, we will look for an implicit rather than an iterative solution of eq. (68). For all background fields except the moduli turned off, such a solution has been found in ref. [17] assuming the relation $\gamma_{i j}=F_{i}^{m} F_{j}^{n} \hat{g}_{m n}$. As noted earlier, however, this relation is really incomplete and should be supplemented with the other terms in eq. (51). Then, even in the pure moduli case, an additional term from the external space arises so that $\gamma_{i j}=F_{i}^{m} F_{j}^{n} \hat{g}_{m n}+\partial_{i} X^{\mu} \partial_{j} X^{\nu} \eta_{\mu \nu}$ where $\eta_{\mu \nu}$ is the Minkowski metric of the external space. Unfortunately, in the presence of this extra term the solution of ref. [17 no longer works. How, then, can we proceed with the most general background when even the pure moduli case poses such problems? The only systematic way out of this difficulty which we could find is to rewrite eq. (68) in a "full index range" form as

$$
\epsilon^{i j k} V_{j m N} \partial_{k} X^{N}=-\sqrt{-\gamma} \gamma^{i j} \partial_{j} X^{N} \hat{g}_{m N}
$$

where we have defined

$$
V_{j m N}=\frac{1}{2} \hat{b}_{m R N} \partial_{j} X^{R}-2 \partial_{j} Y_{m N}
$$

and $F_{i}^{m}$ has been replaced by $\partial_{i} X^{m}$. This equivalent form is better adapted to the structure of the $\gamma_{i j}$ equation, $\gamma_{i j}=\partial_{i} X^{M} \partial_{j} X^{N} \hat{g}_{M N}$, and has the simple solution

$$
V_{i m N}=-\frac{1}{4 \sqrt{-\gamma}} \epsilon_{i}^{j k} \partial_{j} X^{R} \partial_{k} X^{S} \hat{g}_{m N R S}
$$

with

$$
\hat{g}_{M N R S} \equiv \hat{g}_{M R} \hat{g}_{N S}-\hat{g}_{M S} \hat{g}_{N R} .
$$

The verification is straightforward by inserting (71) into eq. (69) and using the fact that $\gamma_{i j}$ is given by the induced metric (43). Note that is was crucial to rewrite the equation (69) in the "full index range" form (69) to find this solution. Moreover, as can be seen from the definition (70), the off-diagonal dual coordinates $Y_{m \nu}$ fit nicely into this scheme.

Though (71) appears to be the "natural" solution of eq. (69), it is unfortunately not the most general one. Any $Z_{j m N}$ with $\epsilon^{i j k} Z_{j m N} \partial_{k} X^{N}=0$ can be added to $V_{j m N}$ in (71). Clearly, a restriction to the subset of solutions with $Z_{j m N}=0$ can be a source of symmetry breaking (if the subset is noninvariant). Nevertheless, we will proceed with the solution (71) keeping in mind that 
we are actually using a certain subclass of solutions of the dual theory. Later on we will comment on the remaining freedom parameterized by $Z_{j m N}$.

Let us first concentrate on the $(m N)=(m n)$ part of the solution $(71)$, which explicitly reads

$$
V_{i m n}=-\frac{1}{4 \sqrt{-\gamma}} \epsilon_{i}{ }^{j k}\left(\partial_{j} X^{r} \partial_{k} X^{s} \hat{g}_{m n r s}+2 \partial_{j} X^{r} \partial_{k} X^{\sigma} \hat{g}_{m n r \sigma}+\partial_{j} X^{\rho} \partial_{k} X^{\sigma} \hat{g}_{m n \rho \sigma}\right) .
$$

This equation can be easily solved for $\partial_{j} X^{m} \partial_{k} X^{n}$ by inverting $\hat{g}_{m n r s}$ to give

$$
\epsilon^{i j k} \partial_{j} X^{m} \partial_{k} X^{n}=-\sqrt{-\gamma} \gamma^{i j} \hat{g}^{m n r s} V_{j r s}-\frac{1}{2} \epsilon^{i j k} \partial_{j} X^{r} \partial_{k} X^{\sigma} \hat{g}_{r \sigma u v} \hat{g}^{\text {mnuv }}-\frac{1}{4} \epsilon^{i j k} \partial_{j} X^{\rho} \partial_{k} X^{\sigma} \hat{g}_{\rho \sigma u v} \hat{g}^{\text {mnuv }} .
$$

Inserting this expression into equation (68) eliminates the term bilinear in $F_{i}^{m}$, and we get the implicit solution

$$
\begin{aligned}
F_{i}^{m}= & 2 \widetilde{\hat{g}}^{m n} \frac{1}{\sqrt{-\gamma}} \epsilon_{i}{ }^{j k} \partial_{j} Y_{n N} \partial_{k} X^{N}-4 \widetilde{\widehat{b}}^{\bar{n} m} \partial_{i} Y_{\bar{n}}+\widetilde{\hat{g}}^{m}{ }_{\nu} \partial_{i} X^{\nu} \\
& -\widetilde{\widehat{b}}^{m}{ }_{r \sigma} \frac{1}{\sqrt{-\gamma}} \epsilon_{i}^{j k} \partial_{j} X^{r} \partial_{k} X^{\sigma}-\widetilde{\hat{b}}^{m}{ }_{\rho \sigma} \frac{1}{2 \sqrt{-\gamma}} \epsilon_{i}{ }^{j k} \partial_{j} X^{\rho} \partial_{k} X^{\sigma} .
\end{aligned}
$$

Here, we have introduced the notation $\bar{m}=\left[m_{1} m_{2}\right]$ for an antisymmetric pair of internal indices, which turns out to be useful in the following. We define the summation over two of these index pairs to include a factor $1 / 2$, that is

$$
v_{\bar{m}} w^{\bar{m}} \equiv \frac{1}{2} v_{m_{1} m_{2}} w^{m_{1} m_{2}}
$$

for any pair of antisymmetric tensors $v_{\bar{m}}$ and $w^{\bar{m}}$. The "dual" quantities in the above solution (75) are defined by

$$
\begin{aligned}
\widetilde{\hat{g}}_{m n} & =\hat{g}_{m n}+\hat{b}_{m \bar{r}} \hat{g}^{\bar{g} \bar{b}_{n \bar{s}}} \\
\widetilde{\hat{b}}^{\bar{m} n} & =\widetilde{\hat{g}}^{n r} \hat{b}_{r \bar{s}} \hat{g}^{\bar{m} \bar{s}} \\
\widetilde{\widehat{g}}_{\nu}^{m} & =-\widetilde{\widehat{g}}^{m r} \hat{g}_{r \nu}-\widetilde{\hat{b}}^{\bar{r} m} \hat{b}_{\nu \bar{r}} \\
\widetilde{\hat{b}}^{m}{ }_{r \sigma} & =\widetilde{\widehat{g}}^{m s} \hat{b}_{s r \sigma}-\widetilde{\hat{b}}^{\bar{s} m} \hat{g}_{\bar{s} r \sigma} \\
\widetilde{\hat{b}}_{\rho \sigma}^{m} & =\widetilde{\widehat{g}}^{m r} \hat{b}_{\rho \sigma r}-\widetilde{\widehat{b}}^{\bar{s} m} \hat{g}_{\bar{s} \rho \sigma} .
\end{aligned}
$$

It is interesting to observe how close these relations are to their string analogs (23). Basically, some of the indices have just been replaced by antisymmetric index pairs.

These results allow us to find an expression for the Bianchi identity of $\epsilon^{i j k} \partial_{j} X^{m} \partial_{k} X^{n}$ in terms of the dual coordinates by using eq. (74) with $F_{i}^{m}$ replaced via eq. (75). Then, from eqs. (65), (66) the completed pair of conjugate momenta and Bianchi identities reads

$$
\begin{aligned}
2 \epsilon^{i j k} \partial_{j} Y_{m N} \partial_{k} X^{N}=\frac{\partial \mathcal{L}_{x}}{\partial\left(\partial_{i} X^{m}\right)}= & \hat{g}_{m n} \sqrt{-\gamma} \gamma^{i j} \partial_{j} X^{n}+\frac{1}{2} \hat{b}_{m n r} \epsilon^{i j k} \partial_{j} X^{n} \partial_{k} X^{r} \\
& +\hat{g}_{m \nu} \sqrt{-\gamma} \gamma^{i j} \partial_{j} X^{\nu}+\hat{b}_{n r \sigma} \epsilon^{i j k} \partial_{j} X^{r} \partial_{k} X^{\sigma}
\end{aligned}
$$




$$
\begin{aligned}
\epsilon^{i j k} \partial_{j} X^{m_{1}} \partial_{k} X^{m_{2}}=\frac{\partial \mathcal{L}_{y}}{\partial\left(\partial_{i} Y_{m_{1} m_{2}}\right)}= & \widetilde{\hat{g}}^{\bar{m} \bar{n}} 4 \sqrt{-\gamma} \hat{b}_{m \rho \sigma} \epsilon^{i j k} \partial_{j} X^{\rho} \partial_{\bar{n}} X^{\sigma} \\
& +\widetilde{\widetilde{b}}^{\bar{m} n} 2 \epsilon^{\bar{m}}{ }^{i j k} \partial_{j} Y_{n N} \partial_{k} X^{N} \gamma^{i j} \partial_{j} X^{\nu}+\widetilde{\hat{b}}_{r \sigma}^{\bar{m}} \epsilon^{i j k} \partial_{j} X^{r} \partial_{k} X^{\sigma} \\
& +\frac{1}{2} \widetilde{\hat{b}}_{\rho \sigma}^{\bar{m}} \epsilon^{i j k} \partial_{j} X^{\rho} \partial_{k} X^{\sigma} .
\end{aligned}
$$

with the "double indexed" dual quantities defined by

$$
\begin{aligned}
& \widetilde{\widehat{g}}^{\bar{m} \bar{n}}=\hat{g}^{\bar{m} \bar{n}}-\widetilde{\hat{b}}^{\bar{m} r} \widetilde{\hat{g}}_{r s} \widetilde{\hat{b}}^{\bar{n} s} \\
& \widetilde{\hat{g}}_{\nu}^{\bar{m}}=-\widetilde{\hat{b}}^{\bar{m} n} \hat{g}_{n \nu}+\widetilde{\widehat{g}}^{\bar{m}} \bar{n} \widetilde{\hat{b}}_{\bar{n} \nu}
\end{aligned}
$$

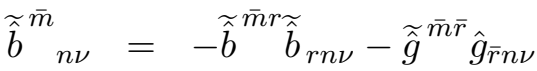

$$
\begin{aligned}
& \widetilde{\hat{b}}_{\rho \nu}^{\bar{m}}=-\widetilde{\hat{b}}^{\bar{m} r} \widetilde{\hat{b}}_{r \rho \nu}-\widetilde{\widehat{g}}^{\bar{m} \bar{r}} \hat{g}_{\bar{r} \rho \nu} \text {. }
\end{aligned}
$$

As in the string case, we would like to solve for the Bianchi identities of $\epsilon^{i j k} \partial_{j} Y_{m N} \partial_{k} X^{N}$ and $\epsilon^{i j k} \partial_{j} X^{m_{1}} \partial_{k} X^{m_{2}}$ which still appear on the right hand sides of the eqs. (78) and (79). This leads to

$$
\begin{aligned}
\left(\begin{array}{c}
2 \epsilon^{i j k} \partial_{j} Y_{m N} \partial_{k} X^{N} \\
\epsilon^{i j k} \partial_{j} X^{m_{1}} \partial_{k} X^{m_{2}}
\end{array}\right)= & \left(\begin{array}{cc}
\widetilde{\hat{g}}_{m n} & \hat{b}_{m \bar{r}} \hat{g}^{\bar{r} \bar{n}} \\
\hat{g}^{\bar{m} \bar{r}} \hat{b}_{n \bar{r}} & \hat{g}^{\bar{m} \bar{n}}
\end{array}\right)\left[\left(\begin{array}{c}
\sqrt{-\gamma} \gamma^{i j} \partial_{j} X^{n} \\
\sqrt{-\gamma} \gamma^{i j} \partial_{j} Y_{\bar{n}}
\end{array}\right)\right. \\
& +\left(\begin{array}{c}
\hat{g}^{n r} \hat{g}_{r \nu} \\
\widetilde{\hat{g}}_{\bar{n} \bar{r}} \widetilde{\hat{g}}_{\nu}^{\bar{r}}
\end{array}\right) \sqrt{-\gamma} \gamma^{i j} \partial_{j} X^{\nu}+\left(\begin{array}{c}
\hat{g}^{n s} \hat{b}_{s r \sigma} \\
\widetilde{\hat{g}}_{\bar{n} \bar{s} \bar{s}}{ }_{r \sigma}
\end{array}\right) \epsilon^{i j k} \partial_{j} X^{r} \partial_{k} X^{\sigma} \\
& +\frac{1}{2}\left(\begin{array}{c}
\hat{g}^{n s} \hat{b}_{s \rho \sigma} \\
\widetilde{\hat{g}}_{\bar{n} \overline{\hat{s}}} \widetilde{\widehat{b}}_{\rho \sigma}^{\bar{s}}
\end{array}\right) \epsilon^{i j k} \partial_{j} X^{\rho} \partial_{k} X^{\sigma}
\end{aligned}
$$

Turning off all background fields except the moduli (which makes the last three terms on the right hand side vanish) leaves us with an equation similar to the one found in ref. [17]. In particular the moduli matrix is identical to the one found there. This means that we are able to arrive at the correct moduli transformations. An explicit example for this will be discussed in the next section. Note, however, that we have derived this result taking the effect of the external space into account. We are, therefore, truly dealing with a membrane in a $\hat{D}=11$-dimensional target space.

To write eq. (81) in a more compact form, we abbreviate

$$
\begin{aligned}
G_{m n} & =\hat{g}_{m n} \\
G_{\bar{m} \bar{n}} & =\hat{g}_{\bar{m} \bar{n}} \\
B_{m \bar{n}} & =\hat{b}_{m \bar{n}} \\
A_{\nu}^{(1) n} & =\hat{g}^{n r} \hat{g}_{r \nu} \\
A_{\nu \bar{n}}^{(2)} & =\widetilde{\widehat{g}}_{\bar{n} \bar{r}} \widetilde{\widehat{g}}_{\nu}^{\bar{r}}=\hat{b}_{\nu \bar{n}}-\hat{b}_{\bar{n} r} A_{\nu}^{(1) r} \\
V_{r \sigma}^{(1) n} & =\hat{g}^{n s} \hat{b}_{s r \sigma}
\end{aligned}
$$




$$
\begin{aligned}
V_{r \sigma \bar{n}}^{(2)} & =\widetilde{\hat{g}}_{\bar{n} \bar{s}} \widetilde{\widehat{b}}_{r \sigma}^{\bar{s}}=-\hat{g}_{\bar{n} r \sigma}-\hat{b}_{\bar{n} s} V_{r \sigma}^{(1) s} \\
B_{\nu \sigma}^{(1) n} & =\hat{g}^{n s} \hat{b}_{s \nu \sigma} \\
B_{\nu \sigma \bar{n}}^{(2)} & =\widetilde{\hat{g}}_{\bar{n} \bar{s} \overline{\hat{b}}}{ }_{\nu \sigma}=-\hat{g}_{\bar{n} \nu \sigma}-\hat{b}_{\bar{n} s} B_{\nu \sigma}^{(1) s} .
\end{aligned}
$$

In addition, we introduce quantities in the $d(d+1) / 2$-dimensional space spanned by $\left(X^{m}, Y_{\bar{m}}\right)$. Let us first consider the background fields. We define the matrix

$$
M=\Phi^{k}\left(\begin{array}{cc}
G_{m n}+B_{m \bar{r}} G^{\bar{r} \bar{s}} B_{n \bar{s}} & B_{m \bar{r}} G^{\bar{r} \bar{n}} \\
G^{\bar{m} \bar{r}} B_{n \bar{r}} & G^{\bar{m} \bar{n}}
\end{array}\right)
$$

which contains the metric and antisymmetric tensor moduli. The $D$-dimensional dilaton $\Phi$ is given by

$$
\Phi=\operatorname{det}\left(G_{m n}\right) .
$$

Its appearance in the above definition with the specific power

$$
k=\frac{1}{D-2}
$$

is purely formal at this point and will be motivated below. The graviphotons and 1-forms from the antisymmetric tensor are grouped into the vectors

$$
\mathbf{A}_{\nu}=\left(\begin{array}{c}
A_{\nu}^{(1) m} \\
A_{\nu \bar{m}}^{(2)}
\end{array}\right), \mathbf{V}_{r \nu}=\Phi^{-k}\left(\begin{array}{c}
V_{r \nu}^{(1) m} \\
V_{r \nu \bar{m}}^{(2)}
\end{array}\right) .
$$

Finally, we introduce the vector

$$
\mathbf{B}_{\nu \sigma}=\left(\begin{array}{c}
B_{\nu \sigma}^{(1) m} \\
B_{\nu \sigma \bar{m}}^{(2)}
\end{array}\right)
$$

which contains the 2 -forms and the graviphotons. Turning to the vertex operators, we define

$$
\begin{aligned}
& \widetilde{\mathcal{F}}=\left(\begin{array}{c}
\widetilde{\mathcal{F}}_{m}^{(1)} \\
\widetilde{\mathcal{F}}^{(2) \bar{m}}
\end{array}\right)=\left(\begin{array}{c}
2 \epsilon^{i j k} \partial_{j} Y_{m N} \partial_{k} X^{N} \\
\epsilon^{i j k} \partial_{j} X^{m_{1}} \partial_{k} X^{m_{2}}
\end{array}\right) \\
& \mathcal{F}=\left(\begin{array}{l}
\mathcal{F}^{(1) m} \\
\mathcal{F}_{\bar{m}}^{(2)}
\end{array}\right)=\Phi^{-k}\left(\begin{array}{c}
\sqrt{-\gamma} \gamma^{i j} \partial_{j} X^{m} \\
4 \sqrt{-\gamma} \gamma^{i j} \partial_{j} Y_{\bar{m}}
\end{array}\right)
\end{aligned}
$$

and

$$
\mathcal{A}^{\nu}=\Phi^{-k} \sqrt{-\gamma} \gamma^{i j} \partial_{j} X^{\nu}, \quad \mathcal{V}^{r \nu}=\epsilon^{i j k} \partial_{j} X^{r} \partial_{k} X^{\nu}, \quad \mathcal{B}^{\nu \sigma}=\epsilon^{i j k} \partial_{j} X^{\nu} \partial_{k} X^{\sigma} .
$$

Then eq. (81) can be written as

$$
\widetilde{\mathcal{F}}=M\left(\mathcal{F}+\mathbf{A}_{\nu} \mathcal{A}^{\nu}+\mathbf{V}_{r \nu} \mathcal{V}^{r \nu}+\frac{1}{2} \mathbf{B}_{\nu \sigma} \mathcal{B}^{\nu \sigma}\right)
$$

This equation is very similar in structure to the corresponding string equation (32). One may therefore expect that $M$ transforms as a tensor, $\widetilde{\mathcal{F}}, \mathcal{F}, \mathbf{A}, \mathbf{V}, \mathbf{B}$ transform as vectors and $\mathcal{A}, \mathcal{V}, \mathcal{B}$ 
transform as singlets under an appropriate representation of the $\mathrm{U}$-duality group in analogy to the string case. To what extent this expectation is really true will be worked out in detail in section 5 . At this point, we will continue to examine the other equations of motion of the theory and attempt to write them in terms of the "covariant" quantities introduced above.

Using the mixed part $(m N)=(m \nu)$ of the solution (71) along with eq. (74), we find for the mixed Bianchi identity (67)

$$
\begin{aligned}
\epsilon^{i j k} \partial_{j} X^{m} \partial_{k} X^{\nu}=\frac{\partial \mathcal{L}_{y}}{\partial\left(\partial_{i} Y_{m \nu}\right)}= & -\hat{g}^{m n} \bar{g}^{\nu \rho}\left[\left(\hat{b}_{n r \rho}-\hat{b}_{n r s} \hat{g}_{\rho}^{s}\right) \sqrt{-\gamma} \gamma^{i j} \partial_{j} X^{r}+\right. \\
& \hat{g}_{\rho}^{r} 4 \sqrt{-\gamma} \gamma^{i j} \partial_{j} Y_{n r}+\left(\hat{b}_{n \sigma \rho}-\hat{g}_{\rho}^{r} \hat{b}_{n \sigma r}\right) \sqrt{-\gamma} \gamma^{i j} \partial_{j} X^{\sigma} \\
& \left.-4 \sqrt{-\gamma} \gamma^{i j} \partial_{j} Y_{n \rho}\right]-\hat{g}_{\rho}^{m} \epsilon^{i j k} \partial_{j} X^{\rho} \partial_{k} X^{\nu}
\end{aligned}
$$

with

$$
\bar{g}_{\mu \nu}=\hat{g}_{\mu \nu}-\hat{g}_{\mu}^{r} \hat{g}_{r \nu}=\hat{g}_{\mu \nu}-A_{\mu}^{(1) r} A_{\nu r}^{(1)}
$$

In the previously introduced short hand notation this equation reads

$$
\begin{aligned}
\mathcal{V}^{m \nu}= & -\Phi^{2 k} \hat{g}^{m n} g^{\nu \rho}\left[A_{\rho n r}^{(2)} \mathcal{F}^{(1) r}+A_{\rho}^{(1) r} \mathcal{F}_{n r}^{(2)}+\left(B_{\rho \sigma n}+\frac{1}{2} A_{\rho}^{(1) r} A_{\sigma n r}^{(2)}\right.\right. \\
& \left.\left.+\frac{1}{2} A_{\sigma}^{(1) r} A_{\rho n r}^{(2)}\right) \mathcal{A}^{\sigma}-\mathcal{J}_{n \rho}\right]-A_{\rho}^{(1) m} \mathcal{B}^{\rho \nu}
\end{aligned}
$$

with

$$
\begin{aligned}
g_{\mu \nu} & =\Phi^{k} \bar{g}_{\mu \nu}=\Phi^{k}\left(\hat{g}_{\mu \nu}-A_{\mu}^{(1) r} A_{\nu r}^{(1)}\right) \\
B_{\mu \nu m} & =\hat{b}_{\mu \nu m}+\frac{1}{2} A_{\mu}^{(1) r} A_{\nu r m}^{(2)}-\frac{1}{2} A_{\nu}^{(1) r} A_{\mu r m}^{(2)}-B_{m r s} A_{\mu}^{(1) r} A_{\nu}^{(1) s}
\end{aligned}
$$

and the vertex operator

$$
\mathcal{J}_{n \rho}=\Phi^{-k} 4 \sqrt{-\gamma} \gamma^{i j} \partial_{j} Y_{n \rho} .
$$

Note, that $g_{\mu \nu}$ in eq. (94) is the metric which naturally appears in the dimensional reduction of the effective theory after the Weyl rescaling. The definition (95) of the 2 -forms is motivated by the string analog (39). Finally, we should find an "appropriate" form of the external conjugate momentum. Using its explicit form (53) and inserting eq. (81) we arrive at

$$
\begin{aligned}
\frac{\partial \mathcal{L}_{x}}{\partial\left(\partial_{i} X^{\mu}\right)}= & g_{\mu \nu} \mathcal{A}^{i \nu}+\left(B_{\nu \mu r}+\frac{1}{2} A_{\nu}^{(1) s} A_{\mu r s}^{(2)}+\frac{1}{2} A_{\mu}^{(1) s} A_{\nu r s}^{(2)}\right) \mathcal{V}^{i r \nu} \\
& +\mathbf{A}_{\mu}^{T} \widetilde{\mathcal{F}}^{i}+\frac{1}{2}\left(\hat{b}_{\mu \nu \rho}-\hat{g}_{\mu}^{r} \hat{b}_{\nu \rho r}\right) \mathcal{B}^{i \nu \rho}
\end{aligned}
$$

We would like to motivate the choice of dilaton powers which we have included into various definitions of background quantities and vertex operators. Clearly, the structure of the membrane equations of motion does not uniquely fix the dilaton powers, which should be chosen in order to get properly transforming quantities. We have therefore used the information from the low energy 
theory that the correct invariant metric after dimensional reduction to $D$ dimensions is the Weyl rescaled metric $g_{\mu \nu}$ in eq. (94) and that the vector fields $\mathbf{A}_{\nu}$ in eq. (86) should not be rescaled (see section 6 for details). This, together with the observation that the vertex operators in $\widetilde{\mathcal{F}}$ and $\mathcal{V}$ should not be rescaled in order to preserve the conservation equations $\partial_{i} \widetilde{\mathcal{F}}^{i}=0$ and $\partial_{i} \mathcal{V}^{i}=0$ essentially fixes the other powers of $\Phi$. In particular, it forces us to include a factor $\Phi^{1 /(D-2)}$ in the definition of $M$, eq. (83). In the next section we will see that this specific power of $\Phi$ is crucial to discover the correct duality symmetry group.

In summary, we have attempted to rewrite the combined equations of motion for $\mathcal{L}_{x}$ and $\mathcal{L}_{y}$ in the most symmetric form, following the string analogy as closely as possible. The result is given by the eqs. (90), (93), (97) along with the definitions (82), (83), (86), (87), (94), (95) for the background fields and (88), (89), (96) for the vertex operators.

\section{Membrane Rotations for Moduli Backgrounds}

In this section, we would like to present an overview of duality symmetries from the membrane worldvolume point of view and their relation to the known U-duality groups of the the low energy supergravity theories in diverse dimensions $D \geq 6$. To keep the discussion as simple as possible, we will, in this section, restrict ourselves to backgrounds with all fields except the moduli turned off. This allows us to point out some of the major problems in a simple setting and motivates why, in the next section, we will concentrate on the case $D=8$ to examine the situation when all background fields are present.

We begin with the observation that the full system of equations of motion and Bianchi identities (for a general background) (90), (93), (97) has a manifest $G L(d)$ symmetry acting on the internal indices $m, n, \ldots$. It is the global part of the internal coordinate transformations and certainly a subgroup of the full duality group G. Let us now attempt to read off this duality group from the moduli part of eq. (90); that is,

$$
\widetilde{\mathcal{F}}=M \mathcal{F}
$$

with $\widetilde{\mathcal{F}}, \mathcal{F}$ and $M$ as defined in eq. (88) and (83). Recall that $\widetilde{\mathcal{F}}, \mathcal{F}$ are $d(d+1) / 2$-dimensional vectors containing vertex operators with the first $d$ entries corresponding to conjugate momenta and the other $d(d-1) / 2$ entries corresponding to Bianchi identities of the original membrane worldvolume theory. The matrix $M$ contains the $d\left(d^{2}+5\right) / 6$ moduli $G_{m n}$ and $B_{m \bar{n}}$. We stress again that eq. (98) has been derived taking the full $\hat{D}=11$ spacetime into account.

We start to explore the invariances of eq. (98) by considering the vertex operator transformations

$$
\widetilde{\mathcal{F}} \rightarrow P \widetilde{\mathcal{F}}, \quad \mathcal{F} \rightarrow P^{-1^{T}} \mathcal{F}
$$

and the moduli transformation

$$
M \rightarrow P M P^{T}
$$


where $P \in \mathbf{G} \subset G L(d(d+1) / 2)$. Clearly, the transformation (100) should preserve the structure of $M$. This restricts the allowed matrices $P$ and determines the group G. A useful general piece of information can be extracted from the formula

$$
\operatorname{det}(M)=\Phi^{\frac{3}{2} \frac{(D-7)(D-8)}{D-2}}
$$

which follows from the definition of $M$, eq. (83). It shows that $\operatorname{det}(M)=1$ for $D=7,8$ so that $P \in \mathbf{G} \subset S L(d(d+1) / 2)$ in these dimensions. Let us now analyze the structure of $M$ case by case.

$\underline{D=10}$ : In this case eq. (98) is a 1 -dimensional equation with

$$
M=\left(\Phi^{9 / 8}\right)
$$

The duality group $\mathbf{G}=G L(1)$ coincides with the internal global coordinate transformations and extends therefore trivially to a symmetry of the full membrane equations (including all background fields). On the level of the effective theory it corresponds to the scaling symmetry of type IIA supergravity.

$\underline{D=9}:$ Eq. (98) is now a 3-component vector equation with the first two components labeled by $m=9,10$ and the third component by an antisymmetric index pair $\bar{m}$. The matrix $M$ contains 3 (metric) moduli. We can use the internal $\epsilon$ tensor $\epsilon^{\bar{m}}$ for a relabeling of basis vectors by contracting it with index pairs $\bar{m}$. Therefore we define modified vertex operators by

$$
\widetilde{\mathcal{F}}=\left(\begin{array}{c}
\widetilde{\mathcal{F}}_{m}^{(1)} \\
\frac{1}{\Phi} \epsilon_{\bar{m}} \widetilde{\mathcal{F}}^{(2) \bar{m}}
\end{array}\right), \quad \mathcal{F}=\Phi^{-1 / 7}\left(\begin{array}{c}
\mathcal{F}^{(1) m} \\
\epsilon^{\bar{m}} \mathcal{F}_{\bar{m}}^{(2)}
\end{array}\right)
$$

For the correspondingly transformed matrix $M$ one finds

$$
M=\left(\begin{array}{cc}
\Phi^{1 / 7} G_{m n} & 0 \\
0 & \Phi^{-6 / 7}
\end{array}\right)
$$

Since $\operatorname{det}(M) \neq 1$ in accordance with the general formula (101) the duality group is $\mathbf{G}=G L(2)$. Again this coincides with the internal global coordinate transformations and therefore extends trivially to the full theory. We observe that the vertex operators $\widetilde{\mathcal{F}}, \mathcal{F}$ transform in the reducible representation $\mathbf{2}+\mathbf{1}$ of $G L(2)$ so that the conjugate momenta in the first two components and the Bianchi identity in the third component do not mix. The matrix $M$ parameterizes the moduli coset $G L(2) / S O(2)$. Clearly, the same $G L(2)$ transformations are found as the U-duality group of $D=9$ supergravity.

$\underline{D=8}$ : Now eq. (98) represents a 6 -component vector equation where the first three entries are labeled by $m=8,9,10$ and the last three components by an antisymmetric pair $\bar{m}$. The moduli space is 7 -dimensional with 6 metric moduli and 1 modulus from the 3 -form. We can apply a 
similar method as in the $D=9$ case and use the internal $\epsilon$ symbol $\epsilon^{m \bar{m}}$ to convert all index pairs $\bar{m}$ into single indices. Then the vertex operators get modified to

$$
\widetilde{\mathcal{F}}=\left(\begin{array}{c}
\widetilde{\mathcal{F}}_{m}^{(1)} \\
\frac{1}{\Phi} \epsilon_{m \bar{m}} \tilde{\mathcal{F}}^{(2) \bar{m}}
\end{array}\right), \quad \mathcal{F}=\Phi^{-1 / 6}\left(\begin{array}{c}
\mathcal{F}^{(1) m} \\
\epsilon^{m \bar{m}} \mathcal{F}_{\bar{m}}^{(2)}
\end{array}\right) \text {. }
$$

Note that now the upper and lower component of $\widetilde{\mathcal{F}}, \mathcal{F}$ have the same index structure. This reflects the earlier mentioned fact that the number of conjugate momenta equals the number of winding modes (of both spatial worldvolume directions) in $D=8$ (and only in $D=8$ ). In the basis (105) the matrix $M$ takes the form

$$
M=M_{2} \otimes M_{3}
$$

with

$$
M_{2}=\Phi^{1 / 2}\left(\begin{array}{cc}
1+\frac{B^{2}}{\Phi} & \frac{B}{\Phi} \\
\frac{B}{\Phi} & \frac{1}{\Phi}
\end{array}\right), \quad M_{3}=\Phi^{-1 / 3}\left(G_{m n}\right) .
$$

Here $B$ is the single modulus from the 3 -form defined by

$$
B_{m \bar{n}}=\frac{B}{\Phi} \epsilon_{m \bar{n}} .
$$

From the tensor structure of $M$, and the fact that $\operatorname{det}\left(M_{2}\right)=\operatorname{det}\left(M_{3}\right)=1$, we learn that the duality group in this case is given by $\mathbf{G}=S L(2) \times S L(3)$. This group is indeed the known Uduality group of $D=8$ supergravity. The matrix $M$ represents a parameterization of the moduli coset $S L(2) / S O(2) \times S L(3) / S O(3)$.

We would like to be more specific about the action of a group element $P \in \mathbf{G}$. We therefore split $P=P_{2} \otimes P_{3}$ with $P_{2}, P_{3}$ in the defining representations of $S L(2), S L(3)$. The two parts of $M$ then transform as

$$
M_{2,3} \rightarrow P_{2,3} M_{2,3} P_{2,3}^{T} .
$$

The action on the vertex operators (105) is described as follows. The $S L(3)$ transformation $P_{3}$ acts on the internal index $m$ in eq. (105), simultaneously for the upper and lower components (the conjugate momenta and Bianchi identities). This part of the group therefore consists of global internal coordinate transformations and extends trivially to the full equations of motion. The situation is quite different for the $S L(2)$ part. It acts on the upper and lower component of $\widetilde{\mathcal{F}}$, $\mathcal{F}$ (for each $m$ in the same way) and therefore exchanges momentum and winding modes of the membrane. In this sense, it appears to be the direct analog of a string $\mathrm{T}$-duality transformation. On the other hand, let us consider the specific $S L(2)$ transformation

$$
S=\left(\begin{array}{cc}
0 & 1 \\
-1 & 0
\end{array}\right) \in S L(2)
$$

and let the 3 -form modulus $B=0$. Then from eq. (109) this transformation acts on the dilaton as

$$
\Phi \stackrel{S}{\rightarrow} \frac{1}{\Phi},
$$


that is, as an S-duality transformation. This confirms the general expectation that $\mathrm{S}$ duality should arise as a momentum/winding-mode exchange on the membrane worldvolume since the dilaton is just a geometrical modulus within the framework of $\mathrm{M}$ theory. We remark that the same $S L(2)$ symmetry, acting on the moduli of the theory, can be found within the framework of the matrix model quantization of M-theory [20]. It is by no means obvious that the $S L(2)$ symmetry can be extended to the full membrane equations of motion including all background fields. This question will be studied in detail in the next section.

$\underline{D=7}$ : Eq. (98) represents a 10-component vector equation with the first four entries indexed by $m=7,8,9,10$ and the others by an antisymmetric pair $\bar{m}$. There are 14 moduli, 10 from the metric and 4 from the antisymmetric tensor field. As in the previous examples, the internal $\epsilon$ tensor can be used to relabel the basis vectors such that the duality symmetry becomes manifest. Since the moduli equation (98) is similar in form to the one found by Duff and $\mathrm{Lu}$ [17, our results for the transformation of $\widetilde{\mathcal{F}}, \mathcal{F}$ and $M$ coincide with the ones given there. We will therefore not give the explicit formulae here, but refer to ref. [17] instead. Let us just mention that the duality group is $G=S L(5)$ which coincides with the U-duality group in $D=7$. Under this group, the vertex operators $\widetilde{\mathcal{F}}, \mathcal{F}$ transform as the second rank antisymmetric tensor representation 10 and $M$ parameterizes the coset $S L(5) / S O(5)$.

$\underline{D=6}$ : In this dimension, for the first time, we encounter a paradox. Eq. (98) is a $15-$ component equation with the first five components indexed by $m=6,7,8,9,10$ and the rest by an antisymmetric pair $\bar{m}$. The known $\mathrm{U}$-duality group in this dimension is $O(5,5)$ and there is obviously no 15 -dimensional representation of this group under which $\widetilde{\mathcal{F}}, \mathcal{F}$ could transform. A resolution of this paradox comes from the observation that, within the framework of the low energy effective action, antisymmetric $\delta$ forms should be Poincaré dualized to $D-\delta-2$ forms if $\delta>(D-2) / 2($ if $\delta=(D-2) / 2$ the $\delta$ form should be paired in the Gaillard-Zumino way [10]) in order to discover the full $\mathrm{U}$-duality group. Therefore, in $D=6$ we should dualize the 3 form $\hat{b}_{\mu \nu \rho}$ to a vector field. Instead of 15 vector fields in $\mathbf{A}_{\nu}$, eq. (86), we are now dealing with 16 which then transform under the spinor representation of $O(5,5)$. Since $\mathbf{A}_{\nu}$ has the same internal index structure as $\mathcal{F}$ and enters the full internal equations of motion (81) in a similar way, it seems natural that eq. (81) should be augmented by one component and should transform as a spinor under $O(5,5)$. Then, also $\widetilde{\mathcal{F}}, \mathcal{F}$ would form spinor representations of $O(5,5)$. Though such a Poincaré dualization is straightforwardly performed in the low energy effective action, it is unclear (to us) how this can be done for the worldvolume theory. Despite the fact that we have no "missing multiplets" (as this phenomenon was called in ref. [17]), since we have taken the full background including $\hat{b}_{\mu \nu \rho}$ into account, we remain unable to find a manifestly $S L(5)$ invariant form of the moduli part in the $D=6$ case. 
It is clear that the problem of "Poincaré dualizing on the worldvolume" also arises in other dimensions and affects more fields the lower the dimension is. However, even in $D=7$ we would have encountered this problem if we had taken the 2 forms $\hat{b}_{\mu \nu m}$ into account. These 2 forms fit into a multiplet of the $D=7 \mathrm{U}$-duality group $S L(5)$ only if they are augmented by the dual of the 3 form $\hat{b}_{\mu \nu \rho}$. In $D=8$ the dualizing problem affects the 3 form $\hat{b}_{\mu \nu \rho}$ only. In the low energy effective action it has to be paired in the Gaillard-Zumino way to form an $S L(2)$ doublet. All other background fields, however, fit into multiplets of the $D=8 \mathrm{U}$-duality group $S L(2) \times S L(3)$ without dualization. It is for this reason that we will concentrate on the $D=8$ example in the next section.

\section{The Example $D=8$ with General Background}

In the previous section, we have discussed how U-duality symmetries can be read off from the moduli part of the worldvolume theory. Here, we would like to generalize this discussion to include the full content of background fields. As we have seen, this generalization is trivial in $D=9,10$ since the U-duality groups in these dimensions coincide with the global internal coordinate transformations. On the other hand, if we decrease the dimension some background fields have to be Poincaré dualized to discover the full U-duality group and, unfortunately, we are generally unable to do this in the worldvolume theory. The "cleanest" case, from this point of view, is the $D=8$ one, as explained in the end of the last section. Given our ignorance on how to perform the dualization explicitly on the worldvolume, we therefore concentrate on the $D=8$ example.

First, we would like to analyze the internal equations of motion, eq. (90), in this case. They read

$$
\widetilde{\mathcal{F}}=M\left(\mathcal{F}+\mathbf{A}_{\nu} \mathcal{A}^{\nu}+\mathbf{V}_{r \nu} \mathcal{V}^{r \nu}+\frac{1}{2} \mathbf{B}_{\nu \sigma} \mathcal{B}^{\nu \sigma}\right)
$$

with the vertex operators $\widetilde{\mathcal{F}}, \mathcal{F}$ and $\mathcal{A}^{\nu}, \mathcal{V}^{r \nu}, \mathcal{B}^{\nu \sigma}$ defined in eq. (88) and (89) and the background $M, \mathbf{A}_{\nu}, \mathbf{V}_{r \nu}$ and $\mathbf{B}_{\nu \sigma}$ defined in eq. (83), (86) and (87). In the previous section, we have already analyzed the moduli part, $\widetilde{\mathcal{F}}=M \mathcal{F}$, of this equation. It turned out that the internal $\epsilon$ symbol $\epsilon^{m \bar{m}}$ should be used to convert antisymmetric index pairs $\bar{m}$ into single indices $m$. The vertex operators relabeled in such a way then read

$$
\widetilde{\mathcal{F}}_{m}=\left(\begin{array}{c}
\widetilde{\mathcal{F}}_{m}^{(1)} \\
\frac{1}{\Phi} \epsilon_{m \bar{m}} \widetilde{\mathcal{F}}^{(2) \bar{m}}
\end{array}\right), \quad \mathcal{F}^{m}=\Phi^{-1 / 6}\left(\begin{array}{c}
\mathcal{F}^{(1) m} \\
\epsilon^{m \bar{m}} \mathcal{F}_{\bar{m}}^{(2)}
\end{array}\right),
$$

where we have made the internal index $m$ explicit in this notation. Correspondingly, the moduli matrix $M$ in this basis takes the form

$$
M=M_{2} \otimes M_{3}
$$


with $M_{2}, M_{3}$ given in eq. (107). Since $\operatorname{det}\left(M_{2}\right)=\operatorname{det}\left(M_{3}\right)=1$ this structure of $M$ determines the group of duality rotations to be $\mathbf{G}=S L(2) \times S L(3)$. A group element $P=P_{2} \otimes P_{3}$ with $P_{2}, P_{3}$ in the defining representation of $S L(2), S L(3)$ acts on $\widetilde{\mathcal{F}}, \mathcal{F}$ as

$$
\widetilde{\mathcal{F}}_{m} \rightarrow\left(P_{3}\right)_{m}^{m^{\prime}} P_{2} \widetilde{\mathcal{F}}_{m^{\prime}}, \quad \mathcal{F}^{m} \rightarrow\left(P_{3}^{-1^{T}}\right)_{m^{\prime}}^{m} P_{2}^{-1^{T}} \mathcal{F}^{m^{\prime}}
$$

and on $M$ as

$$
M_{2,3} \rightarrow P_{2,3} M_{2,3} P_{2,3}^{T}
$$

Since $S L(3)$ is part of the global internal coordinate transformations, $P_{3}$ generally transforms the internal indices. In particular this is true for $\widetilde{\mathcal{F}}, \mathcal{F}$. An $S L(2)$ transformation $P_{2}$, on the other hand, acts on the upper and lower component of $\widetilde{\mathcal{F}}, \mathcal{F}$ (similarly for each $m$ ) and therefore exchanges momentum and winding modes. As we have seen, $S L(2)$ is an S-duality symmetry which, in particular, contains the dilaton transformation $\Phi \rightarrow 1 / \Phi$.

Let us now extend this picture to the other background fields. We begin with the vector fields in $\mathbf{A}_{\nu}, \mathbf{V}_{r \nu}$. In the basis (113) these vectors read

$$
\mathbf{A}_{\nu}^{m}=\left(\begin{array}{c}
A_{\nu}^{(1) m} \\
\epsilon^{m \bar{m}} A_{\nu \bar{m}}^{(2)}
\end{array}\right), \quad \mathbf{V}_{r \nu}^{m}=\Phi^{-1 / 6}\left(\begin{array}{c}
V_{r \nu}^{(1) m} \\
\epsilon^{m \bar{m}} V_{r \nu \bar{m}}^{(2)}
\end{array}\right)
$$

where we have made the $S L(3)$ index $m$ explicit in the notation. Let us first discuss the transformation of the corresponding vertex operators $\mathcal{A}^{\nu}$ and $\mathcal{V}^{r \nu}$. As in the string case, from their index structure, we expect them to be singlets under $S L(2)$. On the other hand, since $\mathcal{V}^{r \nu}$ carries an internal index, it transforms under $S L(3)$. Therefore we start with

$$
\mathcal{A}^{\nu} \rightarrow \mathcal{A}^{\nu}, \quad \mathcal{V}^{r \nu} \rightarrow\left(P_{3}^{-1^{T}}\right)_{r^{\prime}}^{r} \mathcal{V}^{r^{\prime} \nu}
$$

as the $S L(2) \times S L(3)$ transformation law for the vertex operators. Given the transformation of $\mathcal{F}$ in eq. (115), and the structure of the internal equations of motion (112), this forces us to require the following transformations for the vectors $\mathbf{A}_{\nu}^{m}$ and $\mathbf{V}_{r \nu}^{m}$

$$
\mathbf{A}_{\nu}^{m} \rightarrow\left(P_{3}^{-1^{T}}\right)_{m^{\prime}}^{m} P_{2}^{-1 T} \mathbf{A}_{\nu}^{m^{\prime}}, \quad \mathbf{V}_{r \nu}^{m} \rightarrow\left(P_{3}^{-1^{T}}\right)_{m^{\prime}}^{m}\left(P_{3}\right)_{r}^{r^{\prime}} P_{2}^{-1^{T}} V_{r^{\prime} \nu}^{m^{\prime}}
$$

Observe that both vectors are $S L(2)$ doublets so that graviphotons and vector fields from the antisymmetric tensor are rotated into each other. As for the string, $\mathbf{A}_{\nu}^{m}$ and $\mathbf{V}_{r \nu}^{m}$ contain the same degrees of freedom, namely the six vector fields of the theory, and one therefore has to check the consistency of the two transformations (119). From eq. (117) and the definitions (82) we find

$$
\mathbf{V}_{r \nu}^{m}=\epsilon^{m u v} M_{3 r u} M_{3 v n} \epsilon_{2} M_{2} \mathbf{A}_{\nu}^{n}
$$

with

$$
\epsilon_{2}=\left(\begin{array}{cc}
0 & 1 \\
-1 & 0
\end{array}\right)
$$


which is the analog of eq. (36) for the string. Using the transformations (116) for $M$ and (119) for $\mathbf{A}_{\nu}^{m}$, together with $P_{2}^{T} \epsilon_{2} P_{2}=\epsilon_{2}$, shows the consistency of eq. (119). Therefore, the vector field terms in the internal equations of motion (112) (the second and third term on the right hand side) are compatible with the $S L(2) \times S L(3)$ symmetry. The transformations (119) for the vector fields exactly coincide with the ones obtained from the low energy effective action, as we will see in the next section.

Finally, to establish an invariance of the internal equations of motion, we need to consider the vector $\mathbf{B}_{\nu \sigma}$ which, in the basis (113), reads

$$
\mathbf{B}_{\nu \sigma}^{m}=\left(\begin{array}{c}
B_{\nu \sigma}^{(1) m} \\
\epsilon^{m \bar{m}} B_{\nu \sigma \bar{m}}^{(2)}
\end{array}\right) .
$$

The "natural" assumption for the corresponding vertex operator $\mathcal{B}^{i \nu \sigma}=\epsilon^{i j k} \partial_{j} X^{\nu} \partial_{k} X^{\sigma}$ is that it transforms as a singlet under $S L(2) \times S L(3)$. From eq. (112), this requires $\mathbf{B}_{\nu \sigma}^{m}$ to be an $S L(2)$ doublet. On the other hand, let us recall from eq. (82) the definition of $B_{\nu \sigma}^{(1) m}$ and $B_{\nu \sigma \bar{m}}^{(2)}$

$$
\begin{aligned}
B_{\nu \sigma}^{(1) m} & =\hat{g}^{m n} \hat{b}_{n \nu \sigma} \\
B_{\nu \sigma \bar{m}}^{(2)} & =-\hat{g}_{\bar{m} \nu \sigma}-\hat{b}_{n \bar{m}} B_{\nu \sigma}^{(1) n} \\
& =-A_{\nu m_{1}}^{(1)} A_{\sigma m_{2}}^{(1)}+A_{\nu m_{2}}^{(1)} A_{\sigma m_{1}}^{(1)}-\hat{b}_{n \bar{m}} B_{\nu \sigma}^{(1) n}
\end{aligned}
$$

We know already that $A_{\nu m}^{(1)}$ is the upper component of an $S L(2)$ doublet. Therefore, as the second expression above contains bilinears in $A_{\nu m}^{(1)}$, the lower entry $B_{\nu \sigma \bar{m}}^{(2)}$ of the vector (122) cannot transform as part of a doublet in contradiction to our previous assumption. Moreover, the new degrees of freedom in $\mathbf{B}_{\nu \sigma}^{m}$ are the 2 forms $\hat{b}_{n \nu \sigma}$, and it is well known from $D=8$ supergravity that they are $S L(2)$ singlets. The only possible conclusion, at this point, therefore is, that the last term in eq. (112) breaks the $S L(2)$ invariance of the internal equations of motion. One might argue that eq. (112), though the correct equation of motion, is not in an appropriate form to manifestly show the $S L(2)$ invariance. Unfortunately, all our attempts to remove the obstruction by modifying the form of eq. (112) failed. We will comment on these attempts and on possible reasons for the symmetry breaking in the final section 7 .

To complete the picture, we would now like to analyze the other equations of motion as well. Eq. (93) for the mixed Bianchi identity can be written as

$$
\mathcal{V}^{m \nu}=-\left(M_{3}^{-1}\right)^{m n} g^{\nu \rho}\left[\frac{1}{\Phi} \epsilon_{n r s} \mathcal{F}^{r T} \epsilon_{2} \mathbf{A}_{\rho}^{s}+\left(B_{\rho \sigma n}+\frac{1}{2 \Phi} \epsilon_{n r s} \mathbf{A}_{\rho}^{r T} \epsilon_{2} \mathbf{A}_{\sigma}^{s}\right) \mathcal{A}^{\sigma}-\mathcal{J}_{n \rho}\right]-A_{\rho}^{(1) m} \mathcal{B}^{\rho \nu} .
$$

Let us assign the transformation property

$$
\mathcal{J}_{n \sigma} \rightarrow\left(P_{3}\right)_{n}^{n^{\prime}} \mathcal{J}_{n^{\prime} \sigma}
$$


to the vertex operator $\mathcal{J}_{n \sigma}$ and the following transformations to the metric (94) and the 2 forms (95)

$$
\begin{aligned}
g_{\mu \nu} & \rightarrow g_{\mu \nu} \\
B_{\nu \sigma n} & \rightarrow\left(P_{3}\right)_{n}^{n^{\prime}} B_{\nu \sigma n^{\prime}} .
\end{aligned}
$$

Then, with the transformations (100), (118) and (119), eq. (125) is invariant under $S L(2) \times S L(3)$ up to the last term. It is interesting to observe that the symmetry breaking term is again proportional to the vertex operator $\mathcal{B}^{i \nu \sigma}=\epsilon^{i j k} \partial_{j} X^{\nu} \partial_{k} X^{\sigma}$ which is bilinear in the external coordinates $X^{\mu}$. Note that, though we could not read off the correct 2 form transformation law form the internal equations of motion (112), we could do so from eq. (125). As we will verify in the next section, the transformation laws (127) and (128) are indeed correct.

Finally, we analyze the external conjugate momentum (97) which now reads

$$
\begin{aligned}
\frac{\partial \mathcal{L}_{x}}{\partial\left(\partial_{i} X^{\mu}\right)}= & \mathbf{A}_{\mu}^{T} \tilde{\mathcal{F}}^{i}+g_{\mu \nu} \mathcal{A}^{i \nu}+\left(B_{\nu \mu r}+\frac{1}{2 \Phi} \epsilon_{r s t} \mathbf{A}_{\nu}^{s T} \epsilon_{2} \mathbf{A}_{\mu}^{t}\right) \mathcal{V}^{i r \nu} \\
& +\frac{1}{2}\left(\hat{b}_{\mu \nu \rho}-\hat{g}_{\mu}^{r} \hat{b}_{\nu \rho r}\right) \mathcal{B}^{i \nu \rho} .
\end{aligned}
$$

Again, all terms except the one proportional to $\mathcal{B}^{i \nu \sigma}$ are $S L(2) \times S L(3)$ invariant. In eq. (129) this

term is associated with the 3 form $\hat{b}_{\mu \nu \rho}$. Therefore, this symmetry breaking is no surprise, since, as noted earlier, the 3 form has to be paired in the Gaillard-Zumino way to form an $S L(2)$ doublet. Because we did not do this in the worldvolume theory, the obstruction in eq. (129) is an expected one. It is conceivable that the origin of the other two symmetry breaking terms in eq. (112) and (125) is related to this.

To summarize, from the membrane equations of motion (112), (125), (129) in $D=8$ we have read off the $S L(2) \times S L(3)$ transformations which act on the vertex operators as in eq. (115), (118), (126) and on the background fields as in eq. (116), (119), (127), (128). Unfortunately, these transformations do not constitute a symmetry of the equations of motion, but leave all terms except the ones proportional to the vertex operator $\mathcal{B}^{i \nu \sigma}=\epsilon^{i j k} \partial_{j} X^{\nu} \partial_{k} X^{\sigma}$ invariant. Despite this fact, the background field transformations agree exactly with the results from $D=8$ supergravity, as we will show in the next section.

\section{Comparison with $D=8$ Supergravity}

In this section, we would like to verify the background transformation laws as determined from the membrane worldvolume theory. This will be done by comparison with $\hat{D}=11$ supergravity dimensionally reduced to $D=8$ [22]. Though we are mainly interested in the specific dimension $D=8$, the first part of the dimensional reduction procedure will be kept general. 
The bosonic part of the $\hat{D}=11$ supergravity Lagrangian reads 2

$$
\mathcal{L}_{11}=\sqrt{-\hat{g}}\left[\frac{1}{4} \hat{R}-\frac{1}{8 \cdot 4 !} \hat{F}_{M N P Q} \hat{F}^{M N P Q}\right]+\frac{1}{82944} \epsilon^{M_{1} \ldots M_{11}} \hat{F}_{M_{1} \ldots M_{4}} \hat{F}_{M_{5} \ldots M_{8}} \hat{b}_{M_{9} M_{10} M_{11}}
$$

with

$$
\hat{F}=4 \partial_{[M} \hat{b}_{N P Q]}
$$

Our index convention for the dimensional reduction is the same as in the previous sections. We use indices $M, N, P, \ldots=0, \ldots, 10$ for the full space, indices $\mu, \nu, \rho, \ldots=0, \ldots, D-1$ for the external space and indices $m, n, r, \ldots=D, \ldots, 10$ for the internal space. For each of these index types we will need corresponding flat tangent space indices which we denote by $A, B, C, \ldots=0, \ldots, 10$ for the full space, $\alpha, \beta, \gamma, \ldots=0, \ldots, D-1$ for the external space and $a, b, c, \ldots=D, \ldots, 10$ for the internal space. For our purpose, it will be sufficient to consider the non-topological part of the Lagrangian (130); that is,

$$
\mathcal{L}_{0}=\sqrt{-\hat{g}}\left[\frac{1}{4} \hat{R}-\frac{1}{8 \cdot 4 !} \hat{F}_{M N P Q} \hat{F}^{M N P Q}\right] .
$$

Following standard methods for dimensional reduction [21] we use the Ansatz

$$
\hat{e}_{M}^{A}=\left(\begin{array}{cc}
\bar{e}_{\mu}^{\alpha} & A_{\mu}^{(1) n} E_{n}^{a} \\
0 & E_{m}^{a}
\end{array}\right)
$$

for the vielbein $\hat{e}_{M}^{A}$ with $\hat{g}_{M N}=\eta_{A B} \hat{e}_{M}^{A} \hat{e}_{N}^{B}$. The internal and external metrics are defined by

$$
\begin{aligned}
G_{m n} & =\delta_{a b} E_{m}^{a} E_{n}^{b} \\
\bar{g}_{\mu \nu} & =\eta_{\alpha \beta} \bar{e}_{\mu}^{\alpha} \bar{e}_{\nu}^{\beta},
\end{aligned}
$$

respectively. For the total metric $\hat{g}_{M N}$ we then get

$$
\hat{g}_{M N}=\left(\begin{array}{cc}
\bar{g}_{\mu \nu}+A_{\mu r}^{(1)} A_{\nu}^{(1) r} & A_{\mu n}^{(1)} \\
A_{\nu m}^{(1)} & G_{m n}
\end{array}\right) .
$$

The most convenient way to perform the dimensional reduction of the 3 form kinetic term in the Lagrangian (132) is to first express it in terms of the flat field strength $\hat{F}_{A B C D}=\hat{e}_{A}^{M} \hat{e}_{B}^{N} \hat{e}_{C}^{P} \hat{e}_{D}^{Q} \hat{F}_{M N P Q}$, then perform the reduction and finally convert back to curved external indices using $\bar{e}_{\mu}^{\alpha}$. In such a way, and by inserting the above expression for the metric, we arrive at the following Lagrangian

$$
\begin{aligned}
\mathcal{L}_{0}=\sqrt{-\bar{g}} \sqrt{\Phi}[ & \frac{1}{4} \bar{R}+\frac{1}{16} \partial_{\mu} G_{m n} \partial^{\mu} G^{m n}+\frac{1}{16} \Phi^{-2} \partial_{\mu} \Phi \partial^{\mu} \Phi \\
& -\frac{1}{16} G_{m n} F_{\mu \nu}^{(1) m} F^{(1) \mu \nu n}-\frac{1}{8 \cdot 4 !} F_{\mu \nu \rho \sigma} F^{\mu \nu \rho \sigma}-\frac{1}{8 \cdot 3 !} F_{\mu \nu \rho s} F^{\mu \nu \rho s} \\
& \left.-\frac{1}{32} F_{\mu \nu r s} F^{\mu \nu r s}-\frac{1}{8 \cdot 3 !} F_{\mu n r s} F^{\mu n r s}\right]
\end{aligned}
$$

\footnotetext{
${ }^{2}$ We are using the conventions of ref. [6] except for an additional rescaling of the 3 form by a factor $1 / 2$.
} 
with the dilaton

$$
\Phi=\operatorname{det}\left(G_{m n}\right)
$$

and

$$
\begin{aligned}
F_{\mu \nu}^{(1) m} & =\partial_{\mu} A_{\nu}^{(1) m}-\partial_{\nu} A_{\mu}^{(1) m} \\
F_{\mu \nu \rho \sigma} & =\bar{e}_{\mu}^{\alpha} \bar{e}_{\nu}^{\beta} \bar{e}_{\rho}^{\gamma} \bar{e}_{\sigma}^{\delta} \hat{e}_{\alpha}^{M} \hat{e}_{\beta}^{N} \hat{e}_{\gamma}^{P} \hat{e}_{\delta}^{Q} \hat{F}_{M N P Q} \\
F_{\mu \nu \rho s} & =\bar{e}_{\mu}^{\alpha} \bar{e}_{\nu}^{\beta} \bar{e}_{\rho}^{\gamma} \hat{e}_{\alpha}^{M} \hat{e}_{\beta}^{N} \hat{e}_{\gamma}^{P} \hat{F}_{M N P s} \\
F_{\mu \nu r s} & =\bar{e}_{\mu}^{\alpha} \bar{e}_{\nu}^{\beta} \hat{e}_{\alpha}^{M} \hat{e}_{\beta}^{N} \hat{F}_{M N r s} \\
F_{\mu n r s} & =\bar{e}_{\mu}^{\alpha} \hat{e}_{\alpha}^{M} \hat{F}_{M n r s} .
\end{aligned}
$$

To get rid of the factor $\sqrt{\Phi}$ on the right hand side on eq. (137), we perform a Weyl rescaling of the external metric $\bar{g}_{\mu \nu}$ to

$$
g_{\mu \nu}=\Phi^{\frac{1}{D-2}} \bar{g}_{\mu \nu}=\Phi^{\frac{1}{D-2}}\left(\hat{g}_{\mu \nu}-A_{\mu}^{(1) r} A_{\nu r}^{(1)}\right) .
$$

Furthermore, we split $\mathcal{L}_{0}$ into a gravitational, a moduli and a form part as

$$
\mathcal{L}_{0}=\mathcal{L}_{\text {gr }}+\mathcal{L}_{\text {moduli }}+\mathcal{L}_{1 \text { forms }}+\mathcal{L}_{2 \text { forms }}+\mathcal{L}_{3 \text { form }}
$$

For these various parts we find

$$
\begin{aligned}
& \mathcal{L}_{\text {gr }}=\sqrt{-g} \frac{1}{4} R \\
& \mathcal{L}_{\text {moduli }}=\sqrt{-g}\left[-\frac{1}{16(D-2)} \Phi^{-2} \partial_{\mu} \Phi \partial^{\mu} \Phi+\frac{1}{16} \partial_{\mu} G_{m n} \partial^{\mu} G^{m n}\right. \\
& \left.-\frac{1}{8 \cdot 3 !} G^{m m^{\prime}} G^{n n^{\prime}} G^{r r^{\prime}} \partial_{\mu} B_{m n r} \partial^{\mu} B_{m^{\prime} n^{\prime} r^{\prime}}\right] \\
& \mathcal{L}_{1 \text { forms }}=\sqrt{-g}\left[-\frac{1}{16} \Phi^{\frac{1}{D-2}} G_{m n} F_{\mu \nu}^{(1) m} F^{(1) \mu \nu n}-\frac{1}{32} \Phi^{\frac{1}{D-2}} F_{\mu \nu r s} F^{\mu \nu r s}\right] \\
& \mathcal{L}_{2 \text { forms }}=\sqrt{-g}\left[-\frac{1}{8 \cdot 3 !} \Phi^{\frac{2}{D-2}} F_{\mu \nu \rho s} F^{\mu \nu \rho s}\right] \\
& \mathcal{L}_{3 \text { form }}=\sqrt{-g}\left[-\frac{1}{8 \cdot 4 !} \Phi^{\frac{3}{D-2}} F_{\mu \nu \rho \sigma} F^{\mu \nu \rho \sigma}\right],
\end{aligned}
$$

where, from eq. (139), the field strengths are given by

$$
\begin{aligned}
F_{\mu \nu}^{(1) m}= & \partial_{\mu} A_{\nu}^{(1) m}-\partial_{\nu} A_{\mu}^{(1) m} \\
F_{\mu \nu r s}= & \hat{F}_{\mu \nu r s}-A_{\mu}^{(1) m} \hat{F}_{m \nu r s}-A_{\nu}^{(1) n} \hat{F}_{\mu n r s} \\
F_{\mu \nu \rho s}= & \hat{F}_{\mu \nu \rho s}-\left(A_{\mu}^{(1) m} \hat{F}_{m \nu \rho s}+2 \text { perm }\right)+\left(A_{\mu}^{(1) m} A_{\nu}^{(1) n} \hat{F}_{m n \rho s}+2 \mathrm{perm}\right) \\
F_{\mu \nu \rho \sigma}= & \hat{F}_{\mu \nu \rho \sigma}-\left(A_{\mu}^{(1) m} \hat{F}_{m \nu \rho \sigma}+3 \text { perm }\right)+\left(A_{\mu}^{(1) m} A_{\nu}^{(1) n} \hat{F}_{m n \rho \sigma}+5 \text { perm }\right) \\
& -\left(A_{\mu}^{(1) m} A_{\nu}^{(1) n} A_{\rho}^{(1) r} \hat{F}_{m n r \sigma}+2 \text { perm }\right) .
\end{aligned}
$$

So far, we have kept the dimension $D$ general. In what follows, we will concentrate on the case $D=8$ to show that the Lagrangian $\mathcal{L}_{0}$ (except $\mathcal{L}_{3}$ form, see the discussion below) is invariant under the 
$S L(2) \times S L(3)$ background transformations which we have read off from the membrane worldvolume theory in the previous section. It turns out that the various parts of $\mathcal{L}_{0}$, as listed in eq. (142), (again except $\mathcal{L}_{3}$ form $)$ are independently invariant under these transformations. Therefore, we discuss each of these parts separately. We start with

$\underline{\mathcal{L}_{\text {gr }}}$ : This part of the Lagrangian only depends on the Weyl rescaled metric $g_{\mu \nu}$, eq. (140), which, according to eqs. (94) and (127) is a singlet under $S L(2) \times S L(3)$.

$\underline{\mathcal{L}_{\text {moduli }}}$ : To show the $S L(2) \times S L(3)$ invariance, we would like to express $\mathcal{L}_{\text {moduli }}$ in terms of the covariantly transforming quantities $M_{2}, M_{3}$ defined in eq. (107). A straightforward computation using these definitions leads to

$$
\mathcal{L}_{\text {moduli }}=\sqrt{-g} \frac{1}{16}\left[\operatorname{tr}\left(\partial_{\mu} M_{2} \partial^{\mu} M_{2}^{-1}\right)+\operatorname{tr}\left(\partial_{\mu} M_{3} \partial^{\mu} M_{3}^{-1}\right)\right],
$$

which is invariant under the $S L(2) \times S L(3)$ transformations (116) and (127).

$\underline{\mathcal{L}_{1 \text { forms }}}:$ This part of the Lagrangian contains the vector fields, the moduli and the external metric. It should be expressible in terms of the vector $\mathbf{A}_{\nu}$ in eq. (117) and the matrix $M=M_{2} \otimes M_{3}$. Indeed, from eq. (117), (107) and (82) we find

$$
\mathcal{L}_{1 \text { forms }}=-\frac{1}{16} \sqrt{-g} \mathbf{F}_{\mu \nu}^{T} M \mathbf{F}^{\mu \nu}
$$

where

$$
\mathbf{F}_{\mu \nu}=\partial_{\mu} \mathbf{A}_{\nu}-\partial_{\nu} \mathbf{A}_{\mu}
$$

is the $S L(2) \times S L(3)$-covariant field strength. This is, in fact, manifestly invariant under the transformations (116), (119) and (127).

$\underline{\mathcal{L}_{2} \text { forms }}:$ This part contains the 2 forms, the vector fields, the moduli and the external metric. We expect the relevant covariant quantities to be $B_{\mu \nu r}$ in eq. (95), $\mathbf{A}_{\nu}$ in eq. (117) and the matrix $M$. After some computation we find that the field strength $F_{\mu \nu \rho s}$ in eq. (143) can be written as

$$
F_{\mu \nu \rho s}=H_{\mu \nu \rho s}-\frac{1}{2}\left(\frac{1}{\Phi} \epsilon_{s m n} \mathbf{A}_{\mu}^{m T} \epsilon_{2} \mathbf{F}_{\nu \rho}^{n}+2 \text { perm }\right)
$$

where

$$
H_{\mu \nu \rho s}=3 \partial_{[\mu} B_{\nu \rho] s}
$$

is the 2 form field strength. The Lagrangian $\mathcal{L}_{2}$ forms then takes the form

$$
\mathcal{L}_{2 \text { forms }}=-\frac{1}{8 \cdot 3 !}\left(M_{3}^{-1}\right)^{r s} F_{\mu \nu \rho r} F_{s}^{\mu \nu \rho} .
$$

Under the transformations (128), (119) and (116) the field strength $F_{\mu \nu \rho s}$ is $S L(2)$-invariant and $S L(3)$-covariant. This shows the invariance of $\mathcal{L}_{2}$ forms. 
$\mathcal{L}_{3 \text { form }}:$ It is well known that this part of the Lagrangian is not invariant under $S L(2)$ by itself. Instead, the $S L(2)$ symmetry shows up as a Gaillard-Zumino duality rotation between the equations of motion and the Bianchi identity of the 3 form. This means that the 4 -form field strength $D_{\mu \nu \rho \sigma}=4 \partial_{[\mu} C_{\nu \rho \sigma]}$ (which consists of $\hat{b}_{\mu \nu \rho}$ and lower degree forms) has to be paired with $\frac{\partial \mathcal{L}_{11}}{\partial D_{\mu \nu \rho \sigma}}$ to form an $S L(2)$ doublet. As we have already pointed out, we do not know how to perform such a Gaillard-Zumino duality transformation of background fields in the worldvolume theory. We were therefore unable to derive the 3 form transformation law from the worldvolume theory. For this reason, there is nothing more to learn for us from the 3-form equations of motion and we refer to ref. [23] for further details.

To conclude, we have verified that all covariant background field quantities and their transformations under $S L(2) \times S L(3)$ in $D=8$, which we could "reasonably" read off from the membrane worldvolume theory, are indeed correct, as they are in agreement with the $S L(2) \times S L(3)$ invariance of the low energy effective theory.

\section{Discussion}

In this paper, we have attempted to derive U-duality symmetries as symmetries of the membrane worldvolume theory. Our method was to rewrite the membrane equations in a manifest covariant form by pairing equations of motion and Bianchi identities of the original and the dual theory. In doing so, we followed the route which leads to the discovery of $\mathrm{T}$ duality as a symmetry of the string worldsheet as closely as possible.

For pure moduli backgrounds and dimensions $D>6$, we could derive the correct U-duality group and moduli coset parameterization in such a way. This generalizes the work of Duff and $\mathrm{Lu}$ [17] and shows that their results can actually be obtained by taking the full 11-dimensional target space into account. Generally, manifest $\mathrm{U}$ duality in a dimensionally reduced theory requires Poincaré dualization of certain background form fields. For $D \leq 6$ this Poincaré dualization, which we do no know how to explicitly carry out in the worldvolume theory, affects the moduli sector of the membrane equations of motion. Therefore, we could not extend our analysis for pure moduli backgrounds to lower dimensions, $D \leq 6$.

If all background fields are included, the need to Poincaré dualize also influences the equations of motion for $D=7,8$. The cleanest, nontrivial case in this respect is the one for $D=8$ with U-duality group $S L(2) \times S L(3)$, since only the 3 form is affected. For our general analysis, we therefore concentrated on this case. It turned out that the membrane equations of motion could "almost" be written in an $S L(2) \times S L(3)$-invariant form. Moreover, we were able to read off the correct $S L(2) \times S L(3)$-covariant background field quantities for all fields except the 3 form. These results have been verified by comparison with $D=8$ supergravity obtained by dimensional 
reduction of $\hat{D}=11$ supergravity. However, in each part of the membrane equations of motion the $S L(2)$ part of the symmetry is obstructed by a term bilinear in the external target space coordinates $X^{\mu}$. Unfortunately, we have no understanding why these terms appear in the internal and mixed equations of motion. Since the symmetry breaking term in the external equations of motion is associated with the 3 form, its origin is possibly related to our ignorance of how to perform the Gaillard-Zumino construction for the 3 form on the worldvolume. It is conceivable that the origin of the other terms is similar. Perhaps this construction cannot be carried out within the membrane theory, but only by combining it with the 5 -brane worldvolume theory (which, after all, contains a 6 form dual to the membrane 3 form). This would imply that $S L(2)$ is not a symmetry of the classical membrane worldvolume theory. Clearly, we are not drawing such a conclusion from our results, since there are other possible sources of symmetry breaking within our approach. In section 3 , we have pointed out that our solution (71) of the dual theory is actually not the most general one. We have, unsuccessfully, attempted to use the remaining freedom in order to remove the obstruction. Still, we cannot exclude the possibility that there exists a more general solution of eq. (69) which leads to fully $S L(2)$ covariant equations of motion. Another problem, pointed out in ref. [24], arises once operators bilinear in target space coordinates are split up into pieces. For example, the operators $\mathcal{F}^{(1) i m}=\Phi^{-k} \sqrt{-\gamma} \gamma^{i j} \partial_{j} X^{m}$ and $\widetilde{\mathcal{F}}^{(2) i \bar{m}}=\epsilon^{i j k} \partial_{j} X^{m_{1}} \partial_{k} X^{m_{2}}$ transform as upper and lower component of an $S L(2)$ doublet, though this seems to contradict the fact that one is basically the square of the other. A related problem arises, once the transformation of the worldvolume metric $\gamma_{i j}$ is taken into account. It can be computed from eq. (51) and the various transformations for the vertex operators and the metric components. This leads to a complicated nonlinear transformation of $\gamma_{i j}$ which seems to be incompatible with the linear transformation of $\mathcal{F}^{(1) i m}$ and $\widetilde{\mathcal{F}}^{(2) i \bar{m}}$. Though these are important issues, which have to be clarified, we feel that they should not be taken too serious. After all, the charges associated with the conserved currents $\left(\frac{\partial \mathcal{L}}{\partial\left(\partial_{i} X^{m}\right)}=\mathcal{F}^{(1) i m} \hat{g}_{m n}+\ldots, \widetilde{\mathcal{F}}^{(2) i \bar{m}}\right)$ do transform as a doublet under $S L(2)$ 16]. And, perhaps even more significantly, despite the obstruction we encounter, we are able to reproduce a large part of the U-duality symmetry structure directly from the worldvolume. We hope that this is a step forward toward a rigorous proof of $\mathrm{U}$-dualities as symmetries of the membrane worldvolume.

Acknowledgments We would like to thank D. Waldram for numerous interesting discussions and M. Duff for a very helpful email conversation. A. L. is supported by a fellowship from Deutsche Forschungsgemeinschaft (DFG). A. L. and B. A. O. are supported in part by DOE under contract No. DE-AC02-76-ER-03071.

\section{References}


[1] P. K. Townsend, Four Lectures on M Theory, Talk given at Summer School in High-energy Physics and Cosmology, Trieste 1996, DAMTP-R-96-58, hep-th/9612121.

[2] B. de Wit, J. Hoppe and H. Nicolai, Nucl. Phys. B305 [FS23] (1988) 545; T. Banks, W. Fischler, S. H. Shenker and L. Susskind, Phys. Rev. D55 (1997) 5112.

[3] J. Hughes, J. Liu and J. Polchinski, Phys. Lett. B180 (1986) 370.

[4] E. Bergshoeff, E. Sezgin and P. K. Townsend, Phys. Lett. B189 (1987) 75; Annals Phys. 185 (1988) 330.

[5] For a review on string solitons see : M. J. Duff, R. R. Khuri and J. X. Lu, Phys. Rep. 259 (1995) 213.

[6] E. Cremmer, B. Julia and J. Scherk, Phys. Lett. B76 (1978) 409; E. Cremmer and B. Julia, Phys. Lett. B80 (1978) 48; Nucl. Phys. B159 (1979) 141; E. Cremmer, LPTENS 81/18, Lectures given at ICTP Spring School Supergravity, Trieste 1981, published in Trieste Supergrav.School 1981:313.

[7] C. M. Hull and P. K. Townsend, Nucl. Phys. B438 (1995) 109.

[8] See for example: A. Giveon, M. Porrati and E. Rabinovici, Phys. Rep. 244 (1994) 77.

[9] M. J. Duff, Nucl. Phys. B335 (1990) 610.

[10] M. K. Gaillard and B. Zumino, Nucl. Phys. B193 (1981) 221.

[11] J. Maharana and J. H. Schwarz, Nucl. Phys. B390 (1993) 3.

[12] T. H. Buscher, Phys. Lett. B194 (1987) 59; Phys. Lett. B201 (1988) 466.

[13] M. J. Duff, Nucl. Phys. B442 (1995) 47.

[14] A. Font, L. Ibáñez, D. Lüst and F. Quevedo, Phys. Lett. B249 (1990) 35; S. J. Rey, Phys. Rev. D43 (1991) 526. A. Sen, Nucl. Phys. B404 (1993) 109; J. H. Schwarz, Dilaton Axion Symmetry, CALT-68-1815, hep-th/9209125.

[15] For a recent review on supermembranes see : M. J. Duff, Supermembranes, Lectures given at TASI 96, CTP-TAMU-61/96, hep-th/9611203.

[16] J. H. Schwarz and A. Sen, Phys. Lett. B312 (1993) 105.

[17] M. J. Duff and J. X. Lu, Nucl. Phys. B347 (1990) 394.

[18] A. Sen, Mod. Phys. Lett. A11 (1996) 827. 
[19] P. K. Townsend, Phys. Lett. B373 (1996) 68.

[20] W. Taylor, Phys. Lett. B394 (1997) 283; Branes, Fluxes and Duality in M(atrix)-Theory, hep-th/9611202.

[21] J. Scherk and J. H. Schwarz, Nucl. Phys. B153 (1979) 61; E. Cremmer, in Supergravity '81, ed. S. Ferrara and J. G. Taylor, Cambridge Univ. Press, Cambridge 1982.

[22] A. Salam and E. Sezgin, Nucl. Phys. B258 (1985) 284.

[23] L. Andrianopoli, R. D'Auria and S. Ferrara, U-Duality and Central Charges in Various Dimensions Revisited, hep-th/9612105.

[24] R. Percacci and E. Sezgin, Mod. Phys. Lett. A10 (1995) 441. 